\title{
WELL POSEDNESS OF OPERATOR VALUED BACKWARD STOCHASTIC RICCATI EQUATIONS IN INFINITE DIMENSIONAL SPACES
}

\author{
Giuseppina Guatteri \\ Dipartimento di Matematica, Politecnico di Milano \\ piazza Leonardo da Vinci 32, 20133 Milano, Italia \\ e-mail: guatteri@mate.polimi.it \\ Gianmario Tessitore \\ Dipartimento di Matematica e Applicazioni, Università di Milano-Bicocca, \\ via R. Cozzi 53 - Edificio U5, 20125 Milano, Italia \\ e-mail: gianmario.tessitore@unimib.it
}

\begin{abstract}
We prove existence and uniqueness of the mild solution of an infinite dimensional, operator valued, backward stochastic Riccati equation. We exploit the regularizing properties of the semigroup generated by the unbounded operator involved in the equation. Then the results will be applied to characterize the value function and optimal feedback law for a infinite dimensional, linear quadratic control problem with stochastic coefficients.
\end{abstract}

Key words. Backward Stochastic Differential Equations in infinite dimensions, Riccati equation, linear quadratic optimal control, Hilbert spaces, stochastic coefficients.

AMS. 93E20, 60H10, 49A60,35R60

\section{INTRODUCTION}

The present paper is concerned with the following infinite dimensional Backward Stochastic Riccati Equation (BSRE)

$$
\left\{\begin{aligned}
-d P_{t} & =\left(A^{\prime} P_{t}+P_{t} A+C_{t}^{\prime} Q_{t}+Q_{t} C_{t}+C_{t}^{\prime} P_{t} C_{t}-P_{t} B_{t} B_{t}^{\prime} P_{t}+S_{t}\right) d t-Q_{t} d W_{t}, \\
P_{T} & =M
\end{aligned}\right.
$$

where $A$ is a self adjoint operator on the Hilbert space $H$ generating the analytic semigroup $\left(e^{t A}\right) ;\left(W_{t}\right)_{t \geq 0}$ is a real valued standard Brownian motion; $\left(B_{t}\right),\left(C_{t}\right),\left(S_{t}\right)$ are operator valued adapted processes. The unknown of the equation is the couple $(P, Q)$ of operator valued adapted processes .

Yong_Zhou

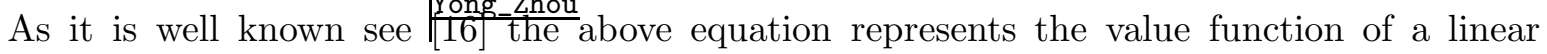
quadratic optimal control problem involving a Hilbert valued state equation with stochastic coefficients (in particular of a control problem with evolution modelled by a parabolic SPDE with stochastic coefficients). It is also well known that, as soon as the solution of the BSRE is obtained, then the synthesis of the optimal control easily follows with a clear applicative interest.

Moreover the special case in which $B_{t} \equiv 0$ (the so called Lyapunov equation) turns out to be essential in the formulation of the Pontyagin maximumprinciple for controlled systems described by stochastic partial differential equations (see [9][10], [4], [5], [6]). This in particular happens in the so called general case in which the space of controls is not convex and the control affects the diffusion term as well (see $\frac{14}{14}$ ). Indeed this is the case in which the second variation process, that satisfies an operator Lyapunov equation, has to be introduced. In this context the research 
on backward evolution equations in spaces of linear operators has gained recently a relevant interest.

The study of BSREs in finite dimensional spaces had quite a long story between the pioneering paper by J.M. Bismut and then S. Peng (see [2 and [13]) and the conclusive paper by S. Tang (see (15) where existence and uniqueness is proved in the most general case.

On the contrary the study of BSREs in infinite dimensional spaces adds specific new difficulties and few results are available. As far as the Lyapunov equation is concerned in the solution is obtained when the final condition $M$ and the forcing term $S$ are Hilbert-Schmidt operators (condition that is rarely satisfied) while in [4], $[6]$ the process $P$ is characterized by an energy equality involving a suitable forward stochastic differential equation in $H$. Finally in 10 the concept of transposed solution is given which again consists in a characterization of $P$ and $Q$ by a suitable duality relation that involves an infinite dimensional forward equation. We notice that in all the above cases no explicit differential or integral equation directly satisfied by $P$ and $Q$ is presented.

Regarding the Riccati equation (that, differently from the Lyapunov equation, is non linear) in 17] we proposed to characterize the $P$-part of the solution using the concept of strong solution which is of common use in PDE theory (see [I] or III). Roughly speaking we characterize the solution as the limit of a sequence of equations with regular (in this case Hilbert-Schmidt) data. This result is good enough to be applied to the corresponding linear quadratic control problem but has the drawback of not saying anything on the martingale term of the solution (the $Q$-term) and consequently not giving the representation through a (differential) equation.

The origin of the difficulties to deal with stochastic backward Riccati (or even Lyapunov) equation in the infinite dimensional case is in the fact that the natural space in which it should be treated is the space $L(H)$ of bounded linear operators in $H$ which is only a Banach space that does not enjoy any of the regularity properties (as UMD or M-type condition) allowing to establish an analogue of the classical Hilbertian stochastic calculus. Moreover although, as we have said above, different characterization of the solution have been recently proposed, it seems to us that the natural notion of solution is the one of mild, solution introduced in the theory of infinite dimensional BSDEs since the seminal paper by 8 . We finally notice that this way both the $P$ and the $Q$ part of the unknown is characterized by a differential equation.

As far as we know this is the first paper in which existence and uniqueness of a mild solution of equation (11.1) is obtained. Indeed we show that $(P, Q)$ is the unique couple of processes (with suitable regularity) verifying

$$
\begin{aligned}
P(t) & =e^{(T-t) A^{\prime}} M e^{(T-t) A}+\int_{t}^{T} e^{(s-t) A^{\prime}} S(s) e^{(s-t) A} d s \\
& +\int_{t}^{T} e^{(s-t) A^{\prime}}\left[C^{\prime}(s) P(s) C(s)+C^{\prime}(s) Q(s)+Q(s) C(s)\right] e^{(s-t) A} d s \\
& +\int_{t}^{T} e^{(s-t) A^{*}} Q(s) e^{(s-t) A} d W(s) \quad \mathbb{P}-\text { a.s. }
\end{aligned}
$$

where $P$ is a predictable process with values in the space of bounded non negative, simmetric, linear operators in $H$ which as we said is, in some sense, the natural space for the equation. On the contrary the identification of the right operators space for the evolution of $Q$ is the main achievement of this work. We shall prove existence and uniqueness of $Q$ as a square-integrable, adapted, process in a space $\mathcal{K}$ of Hilbert-Schimidt operators from suitable domains of the fractional powers of $A$ (see (2..4) $)$. This is an Hilbert space, large enough to contain all bounded operators. This choice will allow to recover stochastic calculus tools. The price to pay is that the term $C_{t}^{\prime} Q_{t}+Q_{t} C+C_{t}^{\prime} P_{t} C_{t}$ becomes unbounded on $\mathcal{K}$. This difficulty will be handled exploiting in a careful (and non completely standard) way the regularizing properties of the semigroup generated by $A$. By the way we have to say that our results rely on the specific properties 
of $A$ that we assume to be self-adjoint with rapidly increasing eigenvalues. Nevertheless our assumptions can cover important classes of strongly elliptic differential operators.

The structure of the propf will be the following: first we introduce suitable approximations of the equation (see (3.30) that can be treated bt the standard Hilbert-Schmidt theory. Then showing the needed convergence estimates we prove existence and uniqueness of the solution to a simplified Lyapunov equation (see 3.44). An a-priori estimate (see (5.3) helps to prove convergence and gives uniqueness. Consequently a fixed point argument vields existence and uniqueness of a solution to the Lyapunov equation. Finally, in Section 遮, we exploit the interplay between the Riccati equation and the corresponding optimal control problem to obtain existence and uniqueness of the mild solution to the BSRE and the synthesis of the optimal control.

We notice that the optimal control problem is given by the following state equation:

$$
\left\{\begin{array}{l}
d y(t)=(A y(t)+B(t) u(t)) d t+C(t) y(t) d W(t) \quad t \in[0, T] \\
y(0)=x
\end{array}\right.
$$

where $y$ is the state of the system and $u$ is the control; $y$ and $u$ are adapted processes with values in $H$, and by the following quadratic cost functional:

$$
\mathbb{E} \int_{0}^{T}\left(\left|\sqrt{S}_{s} y_{s}\right|^{2}+|u(s)|^{2}\right) d s+\mathbb{E}\left\langle M y_{T}, y_{T}\right\rangle
$$

\section{Main Notation and Assumptions}

\section{Some classes of stochastic processes}

Let $G$ be any separable Hilbert space. By $\mathcal{P}$ we denote the predictable $\sigma$-field on $\Omega \times[0, T]$ and by $\mathcal{B}(G)$ Borel $\sigma$-field on $G$. The following classes of processes will be used in this work

- $L_{\mathcal{P}}^{p}(\Omega \times[0, T] ; G), p \in[1,+\infty]$ denotes subset of $L^{p}(\Omega \times[0, T] ; G)$, given by all equivalence classes admitting a predictable version. This space is endowed with the natural norm.

- $C_{\mathcal{P}}\left([0, T] ; L^{p}(\Omega ; G)\right)$ denotes the space of $G$-valued processes $Y$ such that $Y:[0, T] \rightarrow$ $L^{p}(\Omega, G)$ is continuous and $Y$ has a predictable modification, endowed with the norm:

$$
|Y|_{C_{\mathcal{P}}\left([0, T] ; L^{p}(\Omega ; G)\right)}^{p}=\sup _{t \in[0, T]} \mathbb{E}\left|Y_{t}\right|_{G}^{p}
$$

Elements of $C_{\mathcal{P}}\left([0, T] ; L^{p}(\Omega ; G)\right)$ are identified up to modification.

- $L_{\mathcal{P}}^{p}(\Omega ; C([0, T] ; G))$ denotes the space of predictable processes $Y$ with continuous paths in $G$, such that the norm

$$
|Y|_{L_{\mathcal{P}}^{p}(\Omega ; C([0, T] ; G))}^{p}=\mathbb{E} \sup _{t \in[0, T]}\left|Y_{t}\right|_{G}^{p}
$$

is finite. Elements of this space are defined up to indistiguishibility.

Now let us consider the space $L(G)$ of linear and boundedpperators from $G$ to $G$. This space, as long as $G$ is infinite dimensional, is not separable, see [3, pag.23], therefore we introduce the following $\sigma$-field:

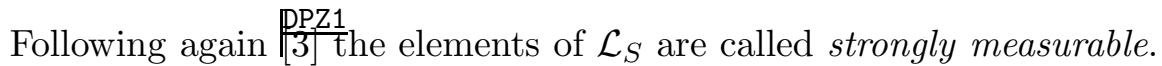

$$
\mathcal{L}_{S}=\{T \in L(G): T u \in A\}, \text { where } u \in G \text { and } A \in \mathcal{B}(G)
$$

We notice that the maps $P \rightarrow|P|_{L(G)}$ and $(P, u) \rightarrow P u$ are measurable from $\left(L(G), \mathcal{L}_{S}\right)$ to $\mathbb{R}$ and from $\left(L(G) \times G, \mathcal{L}_{S} \otimes \mathcal{B}(G)\right)$ to $(G, \mathcal{B}(G))$ respectively.

Moreover $\mathcal{L}_{S}$ is equivalent to the weak $\sigma$-field:

$$
\mathcal{L}_{S}=\{T \in L(G):(T u, x) \in A\}, \text { where } u, x \in G \text { and } A \in \mathcal{B}(\mathbb{R})
$$

We define the following spaces: 
- $L_{\mathcal{P}, S}^{\infty}((0, T) \times \Omega ; L(G))$ a space of predictable processes $Y$ from $(0, T)$ to $L(G)$, endowed with the $\sigma$-field $\mathcal{L}_{S}$. For each element $Y$ there exists a constant $C>0$ such that:

$$
|Y(t, \omega)|_{L(G)} \leq C \quad \mathbb{P}-\text { a.s. for a.e. } t \in(0, T)
$$

In the same way we define $L_{S}^{\infty}\left(\Omega, \mathcal{F}_{T} ; L(G)\right)$ as the space of maps $Y$ from $\left(\Omega, \mathcal{F}_{T}\right)$ into $\left(L(G), \mathcal{L}_{S}\right)$ such that there exists a positive constant $K$ such that:

$$
|Y(\omega)|_{L(G)} \leq K \quad \mathbb{P}-\text { a.s. }
$$

Elements of this space are identified up to modification.

By $\Sigma(G)$ we denote the subspace of all symmetric and operators and by $\Sigma^{+}(G)$ the convex subset of all positive semidefinite operators. We define identically the following spaces: $L_{\mathcal{P}, S}^{\infty}\left((0, T) \times \Omega ; \Sigma^{+}(G)\right), L_{\mathcal{P}, S}^{1}\left((0, T) ; L^{\infty}\left(\Omega, \Sigma^{+}(G)\right)\right)$ and $L_{S}^{\infty}\left(\Omega, \mathcal{F}_{T} ; \Sigma^{+}(G)\right)$.

Setting and general assumptions on the coefficients We fix now an Hilbert space $H$, real and separable, we are going to study the following Lyapunov equation:

$$
\left\{\begin{aligned}
-d P_{t} & =\left(A^{\prime} P_{t}+P_{t} A+C_{t}^{\prime} Q_{t}+Q_{t} C_{t}+C_{t}^{\prime} P_{t} C_{t}\right) d t+S_{t} d t-Q_{t} d W_{t}, \\
P_{T} & =M
\end{aligned}\right.
$$

in the space $L(H)$.

The following assumptions on $A, C, S$ and $M$ will be used throughout the paper:

genhyp Hypothesis 2.1.

A1) $A$ is a self adjoint operator in $H$ and there exist a complete orthonormal basis $\left\{e_{k}: k \geq 1\right\}$ in $H$ (that we fix from now on), a sequence of real numbers $\left\{\lambda_{k}: k \geq 1\right\}$ and $\omega \in \mathbb{R}$, such that

$$
A e_{k}=-\lambda_{k} e_{k}, \quad \text { with } \omega \leq \lambda_{1} \leq \lambda_{2} \leq \cdots \leq \lambda_{k} \leq \ldots,
$$

Moreover we assume that for a suitable $\rho \in\left(\frac{1}{4}, \frac{1}{2}\right)$, it holds

$$
\sum_{k \geq 1} \lambda_{k}^{-2 \rho}<+\infty
$$

Without weakening the generality of the problem we can, and will, assume that $\omega>0$ (just multiply $P$ and $Q$ by an exponential weight) .

As it is well known in this case A generates an analytic semigroup $\left(e^{t A}\right)_{t \geq 0}$ with $\left|e^{t A}\right|_{L(H)} \leq$ 1.

A2) We assume that $C \in L_{\mathcal{P}, S}^{\infty}(\Omega \times[0, T] ; L(H))$. We denote with $M_{C}$ a positive constant such that:

$$
|C(t, \omega)|_{L(H)}<M_{C}, \quad \mathbb{P}-\text { a.s. and for a.e. } t \in(0, T) .
$$

A3) $\left.S \in L_{\mathcal{P}, S}^{\infty}\left((0, T) \times \Omega ; \Sigma^{+}(H)\right)\right)$ and $M \in L_{S}^{\infty}\left(\Omega, \mathcal{F}_{T} ; \Sigma^{+}(H)\right)$.

Remark 2.2. We notice that requirement A.1) in $\frac{\text { genhyp }}{2.1 \mathrm{is}}$ easily fulfilled in the case when $A$ is the realization of the Laplace operator in $H=L^{2}([0, \pi])$ with Dirichlet boundary conditions. One has indeed:

$$
\begin{gathered}
D(A)=H^{2}([0, \pi]) \cap H_{0}^{1}([0, \pi]), \\
e_{k}(x)=(2 / \pi)^{1 / 2} \sin k x, \quad k=1,2, \ldots, \\
\left|\nabla e_{k}(x)\right| \leq(2 / \pi)^{1 / 2} k, \quad k=1,2, \ldots, \\
\lambda_{k}=k^{2}, \quad k=1,2, \ldots
\end{gathered}
$$


Similar considerations can be done for the Laplace operator with Dirichlet boundary conditions on bounded domains of $\mathbb{R}^{n}$.

While requirement A.2) is fulfilled, for instance, as soon as $C(t, \omega)$ is defined on $L^{2}([0, \pi])$ by $(C(t, \omega) x)(\xi):=c(t, \omega, \xi) x(\xi)$, with $c$ any bounded and progressive measurable map $[0, T] \times \Omega \times$ $[0, \pi] \rightarrow \mathbb{R}$. The same holds for A.3), see also section 10 of 17.$]$.

The Hilbertian triple $V \hookrightarrow_{d} H \hookrightarrow_{d} V^{\prime}$

In this paragraph we introduce the Hilbertian triple we will use to build the effective Hilbert space of operators where we are going to solve the Lyapunov equation. Let

$$
V:=D\left((-A)^{\rho}\right)=\left\{x \in H: \sum_{n=1}^{\infty} \lambda_{n}^{2 \rho}\left|\left\langle x, e_{n}\right\rangle\right|^{2}:=|x|_{V}^{2}<\infty\right\} .
$$

By construction $V$ is an Hilbert space endowed with its natural scalar product, in particular $\left\{\lambda_{n}^{-\rho} e_{n}\right\}_{n \geq 1}$ is a complete orthormal basis in $V$.

We can consider also its topological dual $K^{\prime}$ that has the following characterization:

$$
V^{\prime}:=D\left((-A)^{-\rho}\right)
$$

Notice that $V^{\prime}$ is the completion of $H$ with the norm $|\cdot|_{V^{\prime}}^{2}=\sum_{n=1}^{\infty} \lambda_{n}^{-2 \rho}\left|\left\langle x, e_{n}\right\rangle\right|^{2}$ and $\left\{\lambda_{n}^{\rho} e_{n}\right\}_{n \geq 1}$ and that is a complete orthormal basis in $V^{\prime}$.

Once we make the usual identification $H \simeq H^{\prime}$, we have the following dense inclusions:

$$
V \hookrightarrow_{d} H \hookrightarrow_{d} V^{\prime}
$$

We notice that both inclusion operators are Hilbert-Schmidt class

Remark 2.3. Under the previous hypotheses 2.11 we have for all $t>0$

$$
\begin{gathered}
t^{\rho}\left|e^{t A}\right|_{L(H, V)} \leq 1, \quad t^{\rho}\left|e^{t A}\right|_{L\left(V^{\prime}, H\right)} \leq M_{A}, \\
\left|e^{t A}\right|_{L(V)} \leq 1, \quad\left|e^{t A}\right|_{L\left(V^{\prime}\right)} \leq 1
\end{gathered}
$$

The Hilbert space $\mathcal{K}$. We set

$$
\mathcal{K}:=L_{2}(V ; H) \cap L_{2}\left(H ; V^{\prime}\right),
$$

where $L_{2}(V ; H)$ denotes the Hilbert space of Hilbert-Schmidt operators form $V$ to $H$, endowed with the Hilbert-Schmidt norm $|T|_{L_{2}\left(\text { frit }_{1}\right)}=\left(\sum_{i=1}^{\infty}\left|T f_{i}\right|_{H}^{2}\right) \quad\left(\left\{f_{i}: i \in \mathbb{N}\right\}\right.$ being a complete orthonormal basis-b.o.c.-in $V$ ), see [3]. $\mathcal{K}$ will be endowed with the natural norm $|T|_{\mathcal{K}}^{2}=|T|_{L_{2}(V ; H)}^{2}+|T|_{L_{2}(V ; H)}^{2}$

The obvious similar definition holds for $L_{2}\left(H ; V^{\prime}\right)$.

At last we introduce the following subspace of $K$ :

$$
\mathcal{K}_{s}:=\left\{G \in L_{2}(V ; H) \cap L_{2}\left(H ; V^{\prime}\right) \text { such that }\langle G x, y\rangle_{H}=\langle x, G y\rangle_{H} \text { for all } x, y \in V\right\}
$$

We resume its main properties in the following Lemma.

propK Lemma 2.4. The following hold:

(i) $\mathcal{K}$ is a separable Hilbert space,

(ii) $L(H) \subset \mathcal{K}$,

(iii) $T \in \mathcal{K}$ iff $T \in L(V ; H) \cap L\left(H ; V^{\prime}\right)$ and $|T|_{\mathcal{K}}^{2}=\sum_{k=1}^{\infty} \lambda_{k}^{-2 \rho}\left(\left|T e_{k}\right|_{H}^{2}+\left|T^{\prime} e_{k}\right|_{H}^{2}\right)<\infty$, where $T^{\prime} \in L(V ; H) \cap L\left(H ; V^{\prime}\right)$ is the adjoint of $T$ (in the sense that $\langle T v, w\rangle=\left\langle v, T^{\prime} w\right\rangle$. whenever $v \in V$ and $w \in H$ or $w \in V$ and $v \in H$.)

(iv) If $T \in \mathcal{K}_{s}$ then $|T|_{\mathcal{K}_{s}}^{2}=2 \sum_{k=1}^{\infty} \lambda_{k}^{-2 \rho}\left|T e_{k}\right|_{H}^{2}$

Proof. We omit the proof of $(i)$, being obvious.

(ii) Let $G \in L(H)$, then since $\left\{\lambda_{n}^{-\rho} e_{n}\right\}_{n \geq 1}$ is a basis of $V$, we have:

$$
|G|_{L_{2}(V ; H)}=\left(\sum_{n=1}^{\infty} \lambda_{n}^{-2 \rho}\left|G e_{n}\right|_{H}^{2}\right)^{1 / 2} \leq|G|_{L(H)}\left(\sum_{n=1}^{\infty} \lambda_{n}^{-2 \rho}\right)^{1 / 2}
$$


Moreover, recalling that $\left\{e_{n}: n \geq 1\right\}$ is a b.o.c. of $H$, we have:

$$
\begin{aligned}
& |G|_{L_{2}\left(H ; V^{\prime}\right)}=\left(\sum_{n=1}^{\infty}\left|G e_{n}\right|_{V^{\prime}}^{2}\right)^{1 / 2} \leq|G|_{L(H)}\left(\sum_{n=1}^{\infty} \sum_{h=1}^{\infty} \lambda_{h}^{-2 \rho}\left|\left\langle e_{n}, e_{h}\right\rangle\right|_{H}^{2}\right)^{1 / 2} \\
& =|G|_{L(H)}\left(\sum_{h=1}^{\infty} \lambda_{h}^{-2 \rho} \sum_{n=1}^{\infty}\left|\left\langle e_{n}, e_{h}\right\rangle\right|_{H}^{2}\right)^{1 / 2}=|G|_{L(H)}\left(\sum_{h=1}^{\infty} \lambda_{h}^{-2 \rho}\right)^{1 / 2}
\end{aligned}
$$

Thus $G \in \mathcal{K}$.

(iii) Notice that, for any b.o.c. $\left\{f_{k}: k \geq 1\right\}$ of $H$, we have:

$$
\sum_{k=1}^{\infty}\left|T f_{k}\right|_{V^{\prime}}^{2}=\sum_{k=1}^{\infty} \sum_{h=1}^{\infty} \lambda_{h}^{-2 \rho}\left\langle f_{k}, T^{\prime} e_{h}\right\rangle_{H}^{2}=\sum_{h=1}^{\infty} \sum_{k=1}^{\infty} \lambda_{h}^{-2 \rho}\left\langle f_{k}, T^{\prime} e_{h}\right\rangle_{H}^{2}=\sum_{h=1}^{\infty} \lambda_{h}^{-2 \rho}\left|T^{\prime} e_{h}\right|_{H}^{2} .
$$

\section{Mild Solutions of the Lyapunov Equation}

The natural space in which the deterministic Lyapunov equation is studied is the space $\Sigma(H)$ of bounded self adjoint operators in $H$. Unfortunately this is not an Hilbert space and this fact causes serious difficulties when considering stochastic backward differential equations (for instance the essential tool given by the Martingale Representation Theorem does not hold). To overcome this difficulty we will work in the bigger space $\mathcal{K}$ that is a separable Hilbert space.

For convenience we rewrite the equation of interest:

$$
\left\{\begin{aligned}
-d P_{t} & =\left(A^{\prime} P_{t}+P_{t} A+C^{\prime} Q_{t}+Q_{t} C+C^{\prime} P_{t} C\right) d t+S_{t} d t-Q_{t} d W_{t} \\
P_{T} & =M
\end{aligned}\right.
$$

defmild Definition 3.1. A mild solution of problem (K.1) 1s a couple of processes

$$
(P, Q) \in L_{\mathcal{P}, S}^{2}(\Omega, C([0, T] ; \Sigma(H))) \times L_{\mathcal{P}}^{2}\left(\Omega \times[0, T] ; \mathcal{K}_{s}\right)
$$

that solves the following equation, for all $t \in[0, T]$ :

$$
\begin{aligned}
P(t) & =e^{(T-t) A^{\prime}} M e^{(T-t) A}+\int_{t}^{T} e^{(s-t) A^{\prime}} S(s) e^{(s-t) A} d s \\
& +\int_{t}^{T} e^{(s-t) A^{\prime}}\left[C^{\prime}(s) P(s) C(s)+C^{\prime}(s) Q(s)+Q(s) C(s)\right] e^{(s-t) A} d s \\
& +\int_{t}^{T} e^{(s-t) A^{*}} Q(s) e^{(s-t) A} d W(s) \quad \mathbb{P}-\text { a.s. }
\end{aligned}
$$

We first prove an a-priori estimate for mild solutions.

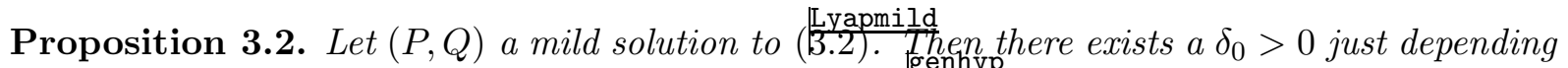
on $T$ and the constants $M_{A}, M_{C}$ and $\rho$ introduced in $\frac{\text { oencyp }}{2.1}$ such that for every $0 \leq \delta \leq \delta_{0}$ the following holds:

$$
|P|_{L^{2}(\Omega ; C([T-\delta, T] ; L(H)))}^{2}+\mathbb{E} \int_{T-\delta}^{T}|Q(s)|_{\mathcal{K}}^{2} d s \leq c\left(\mathbb{E}|M|_{L(H)}^{2}+\delta \mathbb{E} \int_{T-\delta}^{T}|S(s)|_{L(H)}^{2} d s\right) .
$$

where $c$ is a positive constant depending on $\delta_{0}, M_{A}, M_{C}, \rho$ and $T$. 
Proof. Let $(P, Q) \in L_{\mathcal{P}, S}^{2}(\Omega, C([0, T] ; L(H))) \times L_{\mathcal{P}}^{2}\left(\Omega \times[0, T] ; \mathcal{K}_{s}\right)$ be any mild solution, hence we have that:

$$
\begin{aligned}
P(t) & =\mathbb{E}^{\mathcal{F}_{t}}\left[e^{(T-t) A} M e^{(T-t) A}+\int_{t}^{T} e^{(s-t) A} S(s) e^{(s-t) A} d s\right] \\
& +\mathbb{E}^{\mathcal{F}_{t}}\left[\int_{t}^{T} e^{(s-t) A}\left(C^{\prime}(s) P(s) C(s)+C^{\prime}(s) Q(s)+Q(s) C(s)\right) e^{(s-t) A} d s\right] \quad \mathbb{P}-\text { a.s. }
\end{aligned}
$$

We notice that if $(L(t))_{T \geq 0}$ is a Banach space valued process then by Doob's $L^{2}$ inequality

$$
\mathbb{E} \sup _{t \in[r, T]}\left|\mathbb{E}^{\mathcal{F}_{t}} L(t)\right|^{2} \leq \mathbb{E} \sup _{t \in[r, T]}\left[\mathbb{E}^{\mathcal{F}_{t}}\left(\sup _{t \in[r, T]}|L(t)|\right)\right]^{2} \leq 4 \mathbb{E} \sup _{t \in[r, T]}|L(t)|^{2}
$$

Moreover we have:

$$
\begin{aligned}
& \mathbb{E} \sup _{t \in[r, T]}\left|e^{(T-t) A} M e^{(T-t) A}\right|_{L(H)}^{2} \leq|M|_{L(H)}^{2} \\
& \mathbb{E} \sup _{t \in[r, T]}\left|\int_{t}^{T} e^{(s-t) A} C^{\prime}(s) P(s) C(s) e^{(s-t) A} d s\right|_{L(H)}^{2} \leq M_{C}^{4}(T-r) \mathbb{E} \int_{r}^{T}|P(u)|_{L(H)}^{2} d s \\
& \mathbb{E} \sup _{t \in[r, T]}\left|\int_{t}^{T} e^{(s-t) A} S(s) e^{(s-t) A} d s\right|_{L(H)}^{2} \leq(T-r) E \int_{r}^{T}|S(s)|_{L(H)}^{2} d s
\end{aligned}
$$

In estimating the latter terms we notice that even if $G \in \mathcal{K}$ it is not true in general that $G C \in \mathcal{K}$, therefore we have to use the regularity properties of the semigroup (区.t).

$$
\begin{aligned}
& \mathbb{E} \sup _{t \in[r, T]}\left|\int_{t}^{T} e^{(s-t) A}\left[C^{\prime}(s) Q(s)+Q(s) C(s)\right] e^{(s-t) A} d s\right|_{L(H)}^{2} \leq \\
& 2 \mathbb{E}\left\{\sup _{t \in[r, T]}\left[\int_{t}^{T}\left|e^{(s-t) A} C^{\prime}(s) Q(s) e^{(s-t) A}\right|_{L(H)} d s\right]^{2}+\sup _{t \in[r, T]}\left[\int_{t}^{T}\left|e^{(s-t) A} Q(s) C(s) e^{(s-t) A}\right|_{L(H)} d s\right]^{2}\right\}
\end{aligned}
$$

Let us consider the first term:

$$
\begin{aligned}
& \mathbb{E}\left\{\sup _{t \in[r, T]}\left[\int_{t}^{T}\left|e^{(s-t) A} C^{\prime}(s) Q(s) e^{(s-t) A}\right|_{L(H)} d s\right]^{2}\right. \\
& \leq \mathbb{E} \sup _{t \in[r, T]}\left[\int_{t}^{T}\left|e^{(s-t) A}\right|_{L(H)}\left|C^{\prime}(s)\right|_{L(H)}|Q(s)|_{L(V, H)}\left|e^{(s-t) A}\right|_{L(V)} d s\right]^{2} \\
& \leq M_{C}^{2}(T-r) \mathbb{E} \int_{r}^{T}|Q(s)|_{\mathcal{K}}^{2} d s
\end{aligned}
$$

and the second one:

$$
\begin{aligned}
& \mathbb{E} \sup _{t \in[r, T]}\left[\int_{t}^{T}\left|e^{(s-t) A} Q(s) C(s) e^{(s-t) A}\right|_{L(H)} d s\right]^{2} \\
& \leq \mathbb{E} \sup _{t \in[r, T]}\left[\int_{t}^{T}\left|e^{(s-t) A}\right|_{L\left(V^{\prime} ; H\right)}|Q(s)|_{L\left(H ; V^{\prime}\right)}|C(s)|_{L(H)}\left|e^{(s-t) A}\right|_{L(H)} d s\right]^{2} \\
& \leq \mathbb{E} \sup _{t \in[r, T]}\left(\int_{t}^{T} \frac{M_{C}}{(s-t)^{\rho}}|Q(s)|_{\mathcal{K}} d s\right)^{2} \leq M_{C}^{2}(T-r)^{1-2 \rho} \int_{r}^{T}|Q(s)|_{\mathcal{K}}^{2} d s .
\end{aligned}
$$

Summing up all these estimates we obtain that, for $r=T-\delta$ :

$$
\begin{aligned}
& \mathbb{E} \sup _{u \in[T-\delta, T]}|P(u)|_{L(H)}^{2} \\
& \leq C\left(|M|_{L(H)}^{2}+\delta^{2} \mathbb{E} \sup _{u \in[T-\delta, T]}|P(u)|_{L(H)}^{2}+\delta^{1-2 \rho} \mathbb{E} \int_{T-\delta}^{T}|Q(s)|_{\mathcal{K}}^{2} d s+\delta \mathbb{E} \int_{T-\delta}^{T}|S(s)|_{L(H)}^{2} d s\right)
\end{aligned}
$$


where $C$ depends only on $M_{C}, \rho$ and $T$ and for $\delta$ small enough (changing the value of the constant $C)$

$$
\mathbb{E} \sup _{u \in[T-\delta, T]}|P(u)|_{L(H)}^{2} \leq C\left(|M|_{L(H)}^{2}+\delta^{1-2 \rho} \mathbb{E} \int_{T-\delta}^{T}|Q(s)|_{\mathcal{K}}^{2} d s+\delta \mathbb{E} \int_{T-\delta}^{T}|S(s)|_{L(H)}^{2} d s\right)
$$

Now we have to recover an estimate for $Q$, this can not be done in the same, way because the term $Q(s) C(s) \notin \mathcal{K}$, and we can not follow the technique introduced in $\bar{z}$.

Therefore we exploit some duality relation. First of all we multiply both sides by the linear operators $J_{n}:=n(n I-A)^{-1}$.

Such family of operators have the following properties:

(1) $J_{n} e_{k}=\frac{n}{\left(n+\lambda_{k}\right)} e_{k}$, for every $k \geq 1, \quad n \geq 1$,

(2) $\left|J_{n}\right|_{L(H)} \leq 1, \quad\left|J_{n}\right|_{L(V)} \leq 1, \quad\left|J_{n}\right|_{L\left(V^{\prime}\right)} \leq 1$, for every $n \geq 1$,

(3) $\left|J_{n}\right|_{L(H, V)} \leq n^{\rho}, \quad\left|J_{n}\right|_{L\left(V^{\prime}, H\right)} \leq n^{\rho}$

(4) $\lim _{n \rightarrow+\infty} J_{n} x=x$, for every $x \in H$,

(5) $J_{n} \in L_{2}(H)$, for every $n \geq 1$, and $\left|J_{n}\right|_{L_{2}(H)} \leq\left|I_{V, H}\right|_{L_{2}(H)}\left|J_{n}\right|_{L(H, V)}$.

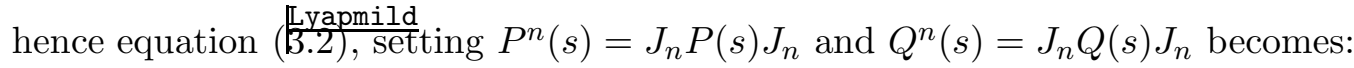

$$
\begin{aligned}
P^{n}(t) & =e^{(T-t) A} J_{n} M J_{n} e^{(T-t) A}+\int_{t}^{T} e^{(s-t) A} J_{n} C^{\prime}(s) P(s) C(s) J_{n} e^{(s-t) A} d s \\
& +\int_{t}^{T} e^{(s-t) A} J_{n} S(s) J_{n} e^{(s-t) A} d s+\int_{t}^{T} e^{(s-t) A}\left[J_{n} C^{\prime}(s) Q(s) J_{n}+J_{n} Q(s) C(s) J_{n}\right] e^{(s-t) A} d s \\
& +\int_{t}^{T} e^{(s-t) A} Q^{n}(s) e^{(s-t) A} d W(s) \quad \mathbb{P}-\text { a.s. }
\end{aligned}
$$

Notice that, thanks to the regularization property of $J_{n},\left(P^{n}, Q^{n}\right) \in L_{\mathcal{P}}^{2}\left(\Omega \times[0, T] ; L_{2}(H)\right) \times$ $L_{\mathcal{P}}^{2}\left(\Omega \times[0, T] ; L_{2}(H)\right)$. In particular

$$
\left|Q_{n}(s)\right|_{L_{2}(H)}^{2} \leq\left|J_{n}\right|_{L\left(V^{\prime} ; H\right)}^{2}|Q(s)|_{\mathcal{K}}^{2}
$$

Moreover $\left(P^{n}, Q^{n}\right)$ is also the unique mild solution of:

$$
\left\{\begin{aligned}
-d P_{t}^{n} & =\left(A^{\prime} P_{t}^{n}+P_{t}^{n} A+C^{\prime} Q_{t}^{n}+Q_{t}^{n} C+C^{\prime} P_{t}^{n} C\right) d t+\hat{S}_{t}^{n} d t-Q_{t}^{n} d W_{t}, \\
P_{T} & =M^{n}
\end{aligned}\right.
$$

where $\hat{S}_{s}^{n}=J_{n} C_{s}^{\prime} P_{s} C_{s} J_{R,}+J_{n} S_{s} J_{n}+J_{n} C_{s}^{\prime} Q_{s} J_{n}+J_{n} Q_{s} C_{s} J_{n} \in L_{\mathcal{P}}^{2}\left(\Omega \times[0, T] ; L_{2}(H)\right)$. We wish

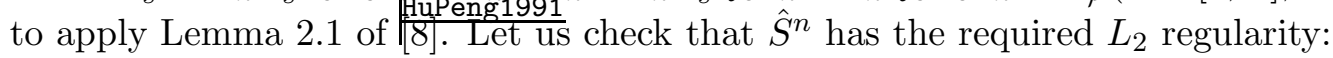

$$
\begin{array}{r}
\mathbb{E} \int_{0}^{T}\left|J_{n} C^{\prime}(s) P(s) C(s) J_{n}\right|_{L_{2}(H)}^{2} d s \leq \mathbb{E} \int_{0}^{T}\left|J_{n}\right|_{L(H)}^{2}\left|C^{\prime}(s)\right|_{L(H)}^{2}|P(s)|_{L(H)}^{2}|C(s)|_{L(H)}^{2}\left|J_{n}\right|_{L_{2}(H)}^{2} d s \\
\leq M_{C}^{4}\left|J_{n}\right|_{L_{2}(H)}^{2}|P|_{L^{2}(\Omega ; C([T-\delta, T] ; L(H)))} \\
\mathbb{E} \int_{0}^{T}\left|J_{n} Q(s) C(s) J_{n}\right|_{L_{2}(H)}^{2} d s \leq \mathbb{E} \int_{0}^{T}\left|J_{n}\right|_{L\left(V^{\prime}, H\right)}^{2}|Q(s)|_{L_{2}\left(H ; V^{\prime}\right)}^{2}|C(s)|_{L(H)}^{2}\left|J_{n}\right|_{L_{2}(H)}^{2} d s \\
\leq n^{2 \rho} M_{C}^{2} \mathbb{E} \int_{0}^{T}|Q(s)|_{\mathcal{K}}^{2} d s
\end{array}
$$




$$
\begin{aligned}
\mathbb{E} \int_{0}^{T}\left|J_{n} C^{\prime}(s) Q(s) J_{n}\right|_{L_{2}(H)}^{2} d s \leq \mathbb{E} \int_{0}^{T}\left|J_{n}\right|_{L(H)}^{2}\left|C^{\prime}(s)\right|_{L(H)}^{2}|Q(s)|_{L_{2}(V ; H)}^{2}\left|J_{n}\right|_{L(H ; V)}^{2} d s \\
\leq n^{2 \rho} M_{C}^{2} \mathbb{E} \int_{0}^{T}|Q(s)|_{\mathcal{K}}^{2} d s .
\end{aligned}
$$

We seek for an estimate independent of $n$ for the martingale term. We are going to use a duality argument, with this purpose we introduce an operator valued process defined as follows

$$
L^{n}(s) e_{k}:=2 \lambda_{k}^{-2 \rho} Q^{n}(s) e_{k}, \quad \text { for } k \geq 1 .
$$

Let us fix $\delta>0$ then consider the following process

$$
X_{t}^{n}=\int_{T-\delta}^{t} e^{(t-s) A} L^{n}(s) e^{(t-s) A} d W(s), \quad t \in[T-\delta, T] .
$$

It can be easily verified that $\left.\left.X^{n} \in C_{\mathbb{p}\left(\mathbb{R}_{1}\right.}-\delta, T\right] ; L^{2}\left(\Omega ; L_{2}(H)\right)\right)$. Therefore by standard regularization arguments, see for instance [3] for the forward equation and 17 for backward equation we can prove that:

$$
\begin{aligned}
& \mathbb{E}\left\langle X^{n}(T), P^{n}(T)\right\rangle_{L_{2}(H)}=\mathbb{E} \int_{T-\delta}^{T}\left\langle L^{n}(s), Q^{n}(s)\right\rangle_{L_{2}(H)} d s-\mathbb{E} \int_{T-\delta}^{T}\left\langle X^{n}(s), J_{n} S(s) J_{n}\right\rangle_{L_{2}(H)} d s \\
& -\mathbb{E} \int_{T-\delta}^{T}\left\langle X^{n}(s), J_{n} C^{\prime}(s) P(s) C(s) J_{n}+J_{n} C^{\prime}(s) Q(s) J_{n}+J_{n} Q(s) C(s) J_{n}\right\rangle_{L_{2}(H)} d s .
\end{aligned}
$$

First of all notice that $\left\langle L^{n}(s), Q^{n}(s)\right\rangle_{L_{2}(H)}=2 \sum_{k=1}^{\infty} \lambda_{k}^{-2 \rho}\left|Q^{n}(s) e_{k}\right|_{H}^{2}$, such quantity corresponds to $\left|Q^{n}\right|_{\mathcal{K}}^{2}$ being $Q^{n}$ a symmetric operator. Thus

$$
\left|\mathbb{E} \int_{T-\delta}^{T}\left\langle L^{n}(s), Q^{n}(s)\right\rangle_{L_{2}(H)} d s\right|=\mathbb{E} \int_{T-\delta}^{T}\left|Q^{n}(s)\right|_{\mathcal{K}}^{2} d s
$$

Let us estimate the process $X_{T}^{n}$, we have for every $t \in[T-\delta, T]$ :

$$
\begin{aligned}
\mathbb{E} \sum_{k \geq 1}\left|X^{n}(t) e_{k}\right|_{H}^{2} \lambda_{k}^{2 \rho} & =\sum_{k \geq 1} \mathbb{E}\left|\int_{T-\delta}^{t} e^{(t-s) A} L^{n}(s) e^{(t-s) A} e_{k} d W_{s}\right|_{H}^{2} \lambda_{k}^{2 \rho} \\
& =\sum_{k \geq 1} \mathbb{E} \int_{T-\delta}^{t} \lambda_{k}^{2 \rho}\left|e^{(t-s) A} L^{n}(s) e^{(t-s) A} e_{k}\right|_{H}^{2} d s \\
& \leq \mathbb{E} \int_{T-\delta}^{T} \sum_{k \geq 1} \lambda_{k}^{-2 \rho} 2\left|Q^{n}(s) e_{k}\right|_{H}^{2} d s=\mathbb{E} \int_{T-\delta}^{T}\left|Q^{n}(s)\right|_{\mathcal{K}}^{2} d s
\end{aligned}
$$

Therefore, using $\frac{\text { stimaxn }}{\left(\frac{3.201}{2.20}\right)}$ with $r=T-\delta$ we have

$$
\begin{aligned}
& \left|\mathbb{E}\left\langle X^{n}(T), P^{n}(T)\right\rangle_{L_{2}(H)}\right| \\
& =\left|\mathbb{E} \sum_{k=1}^{\infty}\left\langle X^{n}(T) e_{k}, P^{n}(T) e_{k}\right\rangle\right| \leq\left(\mathbb{E} \sum_{k=1}^{\infty}\left|X^{n}(T) e_{k}\right|^{2} \lambda_{k}^{2 \rho}\right)^{\frac{1}{2}}\left(\mathbb{E} \sum_{k=1}^{\infty}\left|P^{n}(T) e_{k}\right|^{2} \lambda_{k}^{-2 \rho}\right)^{\frac{1}{2}} \\
& \leq C\left(\mathbb{E} \int_{T-\delta}^{T}\left|Q_{s}^{n}\right|_{\mathcal{K}}^{2} d s\right)^{\frac{1}{2}}\left(\mathbb{E}\left|P^{n}(T)\right|_{L(H)}^{2}\right)^{\frac{1}{2}}
\end{aligned}
$$

Moreover, thanks to $\left(\underline{\text { StimaPUnif }}\right.$ and $\left|P^{n}(T)\right|_{L(H)} \leq|P(T)|_{L(H)}$, we end up with

$$
\begin{aligned}
& \left|\mathbb{E}\left\langle X^{n}(T), P^{n}(T)\right\rangle_{L_{2}(H)}\right| \\
& \leq C\left(\mathbb{E} \int_{T-\delta}^{T}\left|Q_{s}^{n}\right|_{\mathcal{K}}^{2} d s\right)^{\frac{1}{2}}\left(|M|_{L(H)}^{2}+\delta \mathbb{E} \int_{T-\delta}^{T}|S(s)|^{2} d s+\delta^{1-2 \rho} \mathbb{E} \int_{T-\delta}^{T}|Q(s)|_{\mathcal{K}}^{2} d s\right)^{\frac{1}{2}}
\end{aligned}
$$


Regarding $\mathbb{E} \int_{T-\delta}^{T}\left\langle X^{n}(s), J_{n} C^{\prime}(s) Q(s) J_{n}+J_{n} Q(s) C(s) J_{n}\right\rangle_{L_{2}(H)} d s$ we have:

$$
\begin{aligned}
& \left|\mathbb{E} \int_{T-\delta}^{T}\left\langle X^{n}(s), J_{n} C^{\prime}(s) Q(s) J_{n}\right\rangle_{L_{2}(H)} d s\right| \leq M_{C}^{2} \mathbb{E} \int_{T-\delta}^{T}\left(\sum_{k \geq 1}\left|X^{n}(s) e_{k}\right|_{H}^{2} \lambda_{k}^{2 \rho}\right)^{\frac{1}{2}}|Q(s)|_{\mathcal{K}} d s \\
& \leq C \mathbb{E} \int_{T-\delta}^{T}\left(\int_{T-\delta}^{T}\left|Q^{n}(s)\right|_{\mathcal{K}}^{2} d s\right)^{\frac{1}{2}}|Q(s)|_{\mathcal{K}} d s \leq \frac{1}{4} \mathbb{E} \int_{T-\delta}^{T}\left|Q^{n}(s)\right|_{\mathcal{K}}^{2} d s+C \delta \mathbb{E} \int_{T-\delta}^{T}|Q(s)|_{\mathcal{K}}^{2} d s
\end{aligned}
$$

with $C>0$ a constant that may change form line to line but always depends only on the ones introduced in 2.1 . Notice that

$$
\begin{aligned}
& \mathbb{E} \int_{T-\delta}^{T}\left\langle X^{n}(s), J_{n} Q(s) C(s) J_{n}\right\rangle_{L_{2}(H)} d s=\mathbb{E} \int_{T-\delta}^{T} \sum_{k=1}^{\infty}\left\langle X^{n}(s) e_{k}, J_{n} Q(s) C(s) J_{n} e_{k}\right\rangle_{H} d s \\
& =\mathbb{E} \int_{T-\delta}^{T} \sum_{k=1}^{\infty} \sum_{h=1}^{\infty}\left\langle e_{k}, X^{n}(s) e_{h}\right\rangle\left\langle e_{k}, J_{n} C^{\prime}(s) Q(s) J_{n} e_{h}\right\rangle_{H} d s \\
& \leq \mathbb{E} \int_{T-\delta}^{T} \sum_{h=1}^{\infty}\left|X^{n}(s) e_{h}\right|\left|J_{n} C^{\prime}(s) Q(s) J_{n} e_{h}\right| d s \\
& \leq \mathbb{E} \int_{T-\delta}^{T}\left(\sum_{h=1}^{\infty} \lambda_{h}^{2 \rho}\left|X^{n}(s) e_{h}\right|^{2}\right)^{1 / 2}\left(\sum_{h=1}^{\infty} \lambda_{h}^{-2 \rho}\left|Q(s) e_{h}\right|^{2}\right)^{1 / 2} d s .
\end{aligned}
$$

Thus the same conclusion holds, so we have that, by $\frac{\text { (stimaxn }}{(\underline{3.20}):}$

$$
\begin{aligned}
& \left|\mathbb{E} \int_{T-\delta}^{T}\left\langle X^{n}(s), J_{n} C^{\prime}(s) Q(s) J_{n}+J_{n} Q(s) C(s) J_{n}\right\rangle_{L_{2}(H)} d s\right| \\
& \leq \frac{1}{2} \mathbb{E} \int_{T-\delta}^{T}\left|Q^{n}(s)\right|_{\mathcal{K}}^{2} d s+C \delta \mathbb{E} \int_{T-\delta}^{T}|Q(s)|_{\mathcal{K}}^{2} d s
\end{aligned}
$$

Moreover we have that

$$
\begin{aligned}
& \left|\mathbb{E} \int_{T-\delta}^{T}\left\langle X^{n}(s), J_{n} C^{\prime}(s) P(s) C(s) J_{n}\right\rangle_{L_{2}(H)} d s\right| \\
& \leq C \delta|P|_{L_{\mathcal{P}}^{2}(\Omega ; C([T-\delta, T] ; L(H)))}^{2}+\frac{1}{8} \mathbb{E} \int_{T-\delta}^{T}\left|Q^{n}(s)\right|_{\mathcal{K}}^{2} d s,
\end{aligned}
$$

and that, similarly,

$$
\left|\mathbb{E} \int_{T-\delta}^{T}\left\langle X^{n}(s), J_{n} S(s) J_{n}\right\rangle_{L_{2}(H)} d s\right| \leq C \mathbb{E} \int_{T-\delta}^{T}|S(s)|_{L(H)}^{2} d s+\frac{1}{8} \mathbb{E} \int_{T-\delta}^{T}\left|Q^{n}(s)\right|_{\mathcal{K}}^{2} d s,
$$

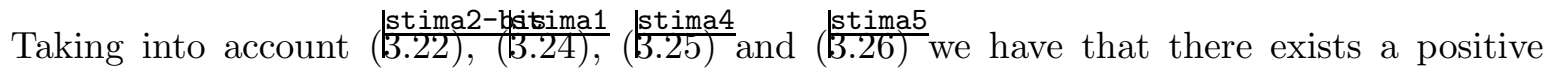
constant $C$ independent of $n$ and $\delta$ such that

$$
\mathbb{E} \int_{T-\delta}^{T}\left|Q^{n}(s)\right|_{\mathcal{K}}^{2} d s \leq C\left(|M|_{L(H)}^{2}+\delta \mathbb{E} \int_{T-\delta}^{T}|S(s)|_{L(H)}^{2} d s+\delta^{1-2 \rho} \mathbb{E} \int_{T-\delta}^{T}|Q(s)|_{\mathcal{K}}^{2} d s\right)
$$

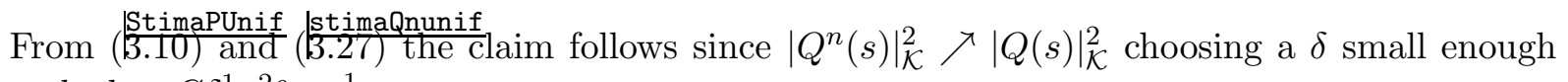
such that $C \delta^{1-2 \rho}<\frac{1}{2}$.

With identical argument we get the estimate in the easier case in which the term $C^{\prime} P C$ is not present 
Remark 3.3. Assume that $Q \in L_{\mathcal{P}}^{2}\left(\Omega \times[0, T] ; \mathcal{K}_{s}\right)$ and that $P$ given by

$$
\begin{aligned}
P(t) & =e^{(T-t) A^{\prime}} M e^{(T-t) A}+\int_{t}^{T} e^{(s-t) A^{\prime}} S(s) e^{(s-t) A} d s \\
& +\int_{t}^{T} e^{(s-t) A^{\prime}}\left[C^{\prime}(s) Q(s)+Q(s) C(s)\right] e^{(s-t) A} d s+\int_{t}^{T} e^{(s-t) A^{*}} Q(s) e^{(s-t) A} d W(s) \quad \mathbb{P}-\text { a.s. }
\end{aligned}
$$

is an adapted $\mathcal{K}$-valued process.

Then there exists a $\delta_{0}>0$ just depending on $T$ and the constants $M_{C}$ and $\rho$ introduced in [2.1 such that for every $0 \leq \delta \leq \delta_{0}$ the following holds:

$$
|P|_{L^{2}(\Omega ; C([T-\delta, T] ; L(H)))}^{2}+\mathbb{E} \int_{T-\delta}^{T}|Q(s)|_{\mathcal{K}}^{2} d s \leq c\left(\mathbb{E}|M|_{L(H)}^{2}+\delta \mathbb{E} \int_{T-\delta}^{T}|S(s)|_{L(H)}^{2} d s\right) .
$$

with $c$ is a positive constant depending on $\delta_{0}, M_{C}, \rho$ and $T$.

We are now in a position to prove existence and uniqueness of the solution to the mild Lyapunov equation

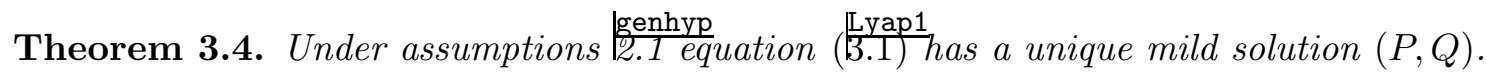

Proof. The idea is classical: we will buid a map $\Gamma$ from the space $L_{\mathcal{P}}^{2}(\Omega, C([0, T] ; H))$ into its self and prove that is a contraction for small time.

In completing this program we follow three steps.

Step 1: regularization We introduce some regularizing processes in order to define $\hat{P}=$ $\Gamma(P)$ for an arbitrary $P \in L_{\mathcal{P}}^{2}(\Omega, C([0, T] ; \Sigma(H)))$. So we fix $P$ and for every $n \geq 1$ we consider the following problem: find $\hat{P}^{n}, \hat{Q}^{n}$ such that

$$
\begin{aligned}
\hat{P}^{n}(t) & =e^{(T-t) A} J_{n} M J_{n} e^{(T-t) A}+\int_{t}^{T} e^{(s-t) A} C^{\prime}(s) J_{n} P(s) J_{n} C(s) e^{(s-t) A} d s \\
& +\int_{t}^{T} e^{(s-t) A} J_{n} S(s) J_{n} e^{(s-t) A} d s+\int_{t}^{T} e^{(s-t) A}\left(C^{\prime}(s) \hat{Q}^{n}(s)+\hat{Q}^{n}(s) C(s)\right) e^{(s-t) A} d s \\
& +\int_{t}^{T} e^{(s-t) A} \hat{Q}^{n}(s) e^{(s-t) A} d W(s) \quad \mathbb{P}-\text { a.s.. }
\end{aligned}
$$

Notice that for every $n \in \mathbb{N}$, we have that $C^{\prime} J_{n} P J_{n} C, J_{n} S J_{n} \in L_{\mathcal{P}}^{2}\left(\Omega \times[0, T] ; L_{2}(H)\right), J_{n} M J_{n} \in$ $L_{2}(H)$. Moreover for every $C \in L(H)$, the map $G \in L_{2}(H) \rightarrow C^{\prime} G+G C \in L_{2}(H)$ is Lipschitz continuous.

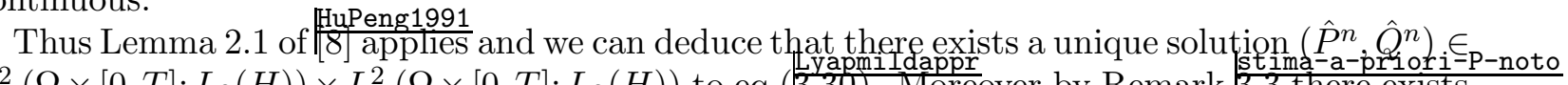
$L_{\mathcal{P}}^{2}\left(\Omega \times[0, T] ; L_{2}(H)\right) \times L_{\mathcal{P}}^{2}\left(\Omega \times[0, T] ; L_{2}(H)\right)$ to eq. (13.30). Moreover by Remark 13.3 there exists $\delta_{0}<1$ small enough and independent of $n$ such that $\forall \delta \leq \delta_{0}$

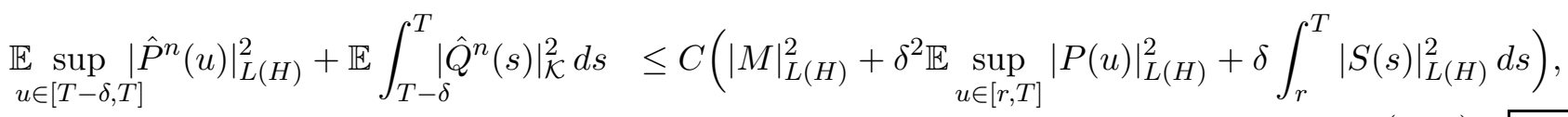

StimaPQn

with $C$ a constant depending only on $M_{C}, T$ and $\rho$ but not on $n$.

We notice here that the operator $P \rightarrow C^{\prime} P C$ is lipschitz from $L_{2}(H)$ to itself as well. We can not treat it as the term $G \rightarrow C^{\prime} G+G C$ since we will then need to lower the regularity of $P$ to the space $\mathcal{K}$ and if $P$ only belonges to $\mathcal{K}$ then the operator $e^{s A} C^{\prime} P C e^{s A}$ is not well defined while $G \rightarrow e^{s A}\left[C^{\prime} G+G C\right] e^{s A}$ is well defined from $\mathcal{K}$ to itself. 
Step 2: limiting procedure Let us evaluate the difference $\hat{P}^{n}-\hat{P}^{m}$ for two integers $m, n$ :

$$
\begin{aligned}
& \hat{P}^{n}(t)-\hat{P}^{m}(t)=e^{(T-t) A} J_{n} M J_{n} e^{(T-t) A}-e^{(T-t) A} J_{m} M J_{m} e^{(T-t) A} \\
& +\int_{t}^{T} e^{(s-t) A}\left(J_{n} S(s) J_{n}-J_{m} S(s) J_{m}\right) e^{(s-t) A} d s \\
& +\int_{t}^{T} e^{(s-t) A} C^{\prime}(s)\left(J_{n} P(s) J_{n}-J_{m} P(s) J_{m}\right) C(s) e^{(s-t) A} d s \\
& +\int_{t}^{T} e^{(s-t) A}\left[C^{\prime}(s)\left(\hat{Q}^{n}(s)-\hat{Q}^{m}(s)\right)+\left(\hat{Q}^{n}(s)-\hat{Q}^{m}(s)\right) C(s)\right] e^{(s-t) A} d s \\
& +\int_{t}^{T} e^{(s-t) A}\left[\hat{Q}^{n}(s)-\hat{Q}^{m}(s)\right] e^{(s-t) A} d W(s) \quad \mathbb{P}-\text { a.s. }
\end{aligned}
$$

we are going to show that

Let's begin to prove ( $\frac{(1 \mathrm{imPn})}{(3.33)}$ by noticing that:

$$
\begin{aligned}
& \lim _{m, n \rightarrow \infty} \mathbb{E} \sup _{t \in[T-\delta, T]}\left|\hat{P}^{n}(t)-\hat{P}^{m}(t)\right|_{\mathcal{K}}^{2}=0 \\
& \lim _{m, n \rightarrow \infty} \mathbb{E} \int_{T-\delta}^{T}\left|\hat{Q}^{n}(s)-\hat{Q}^{m}(s)\right|_{\mathcal{K}}^{2} d s=0
\end{aligned}
$$

$$
\begin{aligned}
& \hat{P}^{n}(t)-\hat{P}^{m}(t)=\mathbb{E}^{\mathcal{F}_{t}}\left(e^{(T-t) A} J_{n} M J_{n} e^{(T-t) A}-e^{(T-t) A} J_{m} M J_{m} e^{(T-t) A}\right) \\
& +\mathbb{E}^{\mathcal{F}_{t}}\left(\int_{t}^{T} e^{(s-t) A}\left(J_{n} S(s) J_{n}-J_{m} S(s) J_{m}\right) e^{(s-t) A} d s\right) \\
& +\mathbb{E}^{\mathcal{F}_{t}}\left(\int_{t}^{T} e^{(s-t) A} C^{\prime}(s)\left(J_{n} P(s) J_{n}-J_{m} P(s) J_{m}\right) C(s) e^{(s-t) A} d s\right) \\
& +\mathbb{E}^{\mathcal{F}_{t}}\left(\int_{t}^{T} e^{(s-t) A}\left[C^{\prime}(s)\left(\hat{Q}^{n}(s)-\hat{Q}^{m}(s)\right)+\left(\hat{Q}^{n}(s)-\hat{Q}^{m}(s)\right) C(s)\right] e^{(s-t) A} d s\right), \quad \mathbb{P}-\text { a.s. }
\end{aligned}
$$

Being $M$ a symmetric operator, we have that

$$
\left|e^{(T-t) A}\left(J_{n} M J_{n}-J_{m} M J_{m}\right) e^{(T-t) A}\right|_{\mathcal{K}}^{2}=\sum_{k=1}^{\infty} \lambda_{k}^{-2 \rho}\left|e^{(T-t) A}\left(J_{n} M J_{n}-J_{m} M J_{m}\right) e^{(T-t) A} e_{k}\right|_{H}^{2}
$$

For every fixed $k \geq 1$ :

$$
\lim _{n, m \rightarrow \infty} \mid\left(\left.J_{n} M\left(J_{n}-J_{m}\right) e_{k}\right|_{H} ^{2}=0, \quad \forall t \in[0, T], \quad \mathbb{P}-\right.\text { a.s. }
$$

and

Moreover

$$
\lim _{n, m \rightarrow \infty}\left|\left(J_{n}-J_{m}\right) M J_{m} e_{k}\right|_{H}^{2}=0, \quad \forall t \in[0, T], \quad \mathbb{P}-\text { a.s. }
$$

$$
\sum_{k=1}^{\infty} \lambda_{k}^{-2 \rho}\left|\left(J_{n} M J_{n}-J_{m} M J_{m}\right) e_{k}\right|_{H}^{2} \leq M_{A}^{4} M_{M}^{2} \sum_{k=1}^{\infty} \lambda_{k}^{-2 \rho}<\infty, \quad \mathbb{P}-\text { a.s. }
$$

Hence by the Dominated Convergence Theorem and the Doob inequality for martingales:

$$
\begin{aligned}
& \quad \lim _{n, m \rightarrow \infty} \mathbb{E}\left[\sup _{t \in[T-\delta, T]}\left|\mathbb{E}^{\mathcal{F}_{t}}\left(e^{(T-t) A}\left(J_{n} M J_{n}-J_{m} M J_{m}\right) e^{(T-t) A}\right)\right|_{\mathcal{K}}^{2}\right] \\
& \leq \lim _{n, m \rightarrow \infty} 4 \mathbb{E}\left|\left(J_{n} M J_{n}-J_{m} M J_{m}\right)\right|_{\mathcal{K}}^{2}=0 .
\end{aligned}
$$

The second and the third term are similar so we'll give the details only of the third. As before we have that for every $k \geq 1$ :

$$
\lim _{n, m \rightarrow \infty} \mid\left(\left.C^{\prime}(s)\left(J_{n} P(s) J_{n}-J_{m} P(s) J_{m}\right) C(s) e_{k}\right|_{H} ^{2}=0, \quad \mathbb{P}-\text { a.s. and for a.e. } s \in[T-\delta, T]\right.
$$


and $\mathbb{P}$-a.s. and for a.e. $s \in[T-\delta, T]$,

$$
\sum_{k \geq 1} \lambda_{k}^{-2 \rho} \mid\left(\left.C^{\prime}(s)\left(J_{n} P(s) J_{n}-J_{m} P(s) J_{m}\right) C(s) e_{k}\right|_{H} ^{2} d s \leq M_{C}^{4} \sum_{k \geq 1} \lambda_{k}^{-2 \rho}<\infty .\right.
$$

Therefore again by the Dominated Convergence Theorem and the Doob inequality for martingales:

$$
\begin{aligned}
& \lim _{n, m \rightarrow \infty} \mathrm{E} \sup _{t \in[T-\delta, T]} \mid \mathbb{E}^{\mathcal{F}_{t}}\left(\left.\int_{t}^{T} e^{(s-t) A}\left[\left(C^{\prime}(s)\left(J_{n} P(s) J_{n}-J_{m} P(s) J_{m}\right) C(s)\right] e^{(s-t) A} d s\right)\right|_{\mathcal{K}} ^{2}\right. \\
& \leq \delta \lim _{n, m \rightarrow \infty} 4 \mathbb{E} \int_{T-\delta}^{T} \sum_{k \geq 1} \lambda_{k}^{-2 \rho} \mid\left(\left.C^{\prime}(s)\left(J_{n} P(s) J_{n}-J_{m} P(s) J_{m}\right) C(s) e_{k}\right|_{H} ^{2} d s=0 .\right.
\end{aligned}
$$

At last let us consider the term

$$
\mathbb{E} \sup _{t \in[T-\delta, T]}\left|\mathbb{E}^{\mathcal{F}_{t}}\left(\int_{t}^{T} e^{(s-t) A}\left[C^{\prime}(s)\left(\hat{Q}^{n}(s)-\hat{Q}^{m}(s)\right)+\left(\hat{Q}^{n}(s)-\hat{Q}^{m}(s)\right) C(s)\right] e^{(s-t) A} d s\right)\right|_{\mathcal{K}}^{2}
$$

First of all:

$$
\begin{aligned}
& \left(\int_{t}^{T}\left|e^{(s-t) A}\left(\hat{Q}^{n}(s)-\hat{Q}^{m}(s)\right) C(s) e^{(s-t) A}\right| \mathcal{K} d s\right)^{2} \\
& =\left[\int_{t}^{T}\left(\sum_{k \geq 1} \lambda_{k}^{-2 \rho}\left|e^{(s-t) A}\left(\hat{Q}^{n}(s)-\hat{Q}^{m}(s)\right) C(s) e^{(s-t) A} e_{k}\right|_{H}^{2}\right)^{1 / 2} d s\right]^{2} \\
& \leq\left(\int_{t}^{T}\left|e^{(s-t) A}\right|_{L\left(V^{\prime} ; H\right)}\left|\left(\hat{Q}^{n}(s)-\hat{Q}^{m}(s)\right)\right|_{L_{2}\left(H ; V^{\prime}\right)}\left(\sum_{k \geq 1} \lambda_{k}^{-2 \rho}\left|C(s) e^{(s-t) A} e_{k}\right|_{H}^{2}\right)^{1 / 2} d s\right)^{2} \\
& \leq M_{C}^{2}\left(\sum_{k \geq 1} \lambda_{k}^{-2 \rho}\right) \int_{t}^{T}(s-t)^{-2 \rho} d s \int_{t}^{T}\left|\hat{Q}^{n}(s)-\hat{Q}^{m}(s)\right|_{\mathcal{K}}^{2} d s \\
& \leq C \delta^{1-2 \rho} \int_{T-\delta}^{T}\left|\hat{Q}^{n}(s)-\hat{Q}^{m}(s)\right|_{\mathcal{K}}^{2} d s
\end{aligned}
$$

Similarily

$$
\begin{aligned}
& {\left[\int_{t}^{T}\left(\sum_{k \geq 1} \lambda_{k}^{-2 \rho}\left|e^{(s-t) A} C^{\prime}(s)\left(\hat{Q}^{n}(s)-\hat{Q}^{m}(s)\right) e^{(s-t) A} e_{k}\right|_{H}^{2}\right)^{1 / 2} d s\right]^{2}} \\
& \leq\left(\int_{t}^{T}\left|e^{(s-t) A}\right|_{L(H)}\left|C^{\prime}(s)\right|_{L(H)}^{2}\left(\sum_{k \geq 1} \lambda_{k}^{-2 \rho} e^{-2(s-t) \lambda_{k}}\left|\left(\hat{Q}^{n}(s)-\hat{Q}^{m}(s)\right) e_{k}\right|_{H}^{2}\right)^{1 / 2} d s\right)^{2} \\
& \leq M_{C}^{2} \delta \int_{t}^{T}\left|\hat{Q}^{n}(s)-\hat{Q}^{m}(s)\right|_{\mathcal{K}}^{2} d s \\
& \leq C \delta^{1-2 \rho} \int_{T-\delta}^{T}\left|\hat{Q}^{n}(s)-\hat{Q}^{m}(s)\right|_{\mathcal{K}}^{2} d s
\end{aligned}
$$

Hence:

$$
\begin{aligned}
& \mathbb{E} \sup _{t \in[T-\delta, T]}\left|\hat{P}^{n}(t)-\hat{P}^{m}(t)\right|_{\mathcal{K}}^{2} \leq C\left[\delta^{1-2 \rho} \int_{T-\delta}^{T} \mathbb{E}\left|\hat{Q}^{n}(s)-\hat{Q}^{m}(s)\right|_{\mathcal{K}}^{2} d s+\mathbb{E}\left|\left(J_{n} M J_{n}-J_{m} M J_{m}\right)\right|_{\mathcal{K}}^{2}\right. \\
& \left.\left.+\delta \mathbb{E} \int_{T-\delta}^{T}\left|C^{\prime}(s)\left(J_{n} P(s) J_{n}-J_{m} P(s) J_{m}\right) C(s)\right|_{\mathcal{K}}^{2} d s+\mathbb{E} \int_{T-\delta}^{T} \mid J_{n} S(s) J_{n}-J_{m} S(s) J_{m}\right)\left.\right|_{\mathcal{K}} ^{2} d s\right] \\
& \leq C \delta^{1-2 \rho} \mathbb{E} \int_{T-\delta}^{T}\left|\hat{Q}^{n}(s)-\hat{Q}^{m}(s)\right|_{\mathcal{K}}^{2} d s+R(m, n)
\end{aligned}
$$


with $R(m, n) \rightarrow 0$ as $m, n \rightarrow+\infty$.

The duality relation between $\hat{P}^{n}-\hat{P}^{m}$ and $\hat{X}^{n}-\hat{X}^{m}$ yields to:

$$
\begin{aligned}
& \mathbb{E}\left\langle\hat{X}^{n}(T)-\hat{X}^{m}(T), \hat{P}^{n}(T)-\hat{P}^{m}(T)\right\rangle_{L_{2}(H)}=\mathbb{E} \int_{T-\delta}^{T}\left\langle\hat{L}^{n}(s)-\hat{L}^{m}(s), \hat{Q}^{n}(s)-\hat{Q}^{m}(s)\right\rangle_{L_{2}(H)} d s \\
& -\mathbb{E} \int_{T-\delta}^{T}\left\langle\hat{X}^{n}(s)-\hat{X}^{m}(s), J_{n} S(s) J_{n}-J_{m} S(s) J_{m}\right\rangle_{L_{2}(H)} d s \\
& -\mathbb{E} \int_{T-\delta}^{T}\left\langle\hat{X}^{n}(s)-\hat{X}^{m}(s), C^{\prime}(s) J_{n} P(s) J_{n} C(s)-C^{\prime}(s) J_{m} P(s) J_{m} C(s)\right\rangle_{L_{2}(H)} d s \\
& -\mathbb{E} \int_{T-\delta}^{T}\left\langle X^{n}(s)-X^{m}(s), C^{\prime}(s)\left(\hat{Q}^{n}(s)-\hat{Q}^{m}(s)\right)+\left(\hat{Q}^{n}(s)-\hat{Q}^{m}(s)\right) C(s)\right\rangle_{L_{2}(H)} d s .
\end{aligned}
$$

where

$$
\mathbb{E} \int_{T-\delta}^{T}\left\langle\hat{L}^{n}(s)-\hat{L}^{m}(s), \hat{Q}^{n}(s)-\hat{Q}^{m}(s)\right\rangle_{L_{2}(H)} d s=\mathbb{E} \int_{T-\delta}^{T}\left|\hat{Q}^{n}(s)-\hat{Q}^{m}(s)\right|_{\mathcal{K}}^{2} d s .
$$

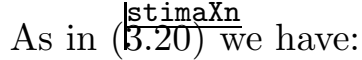

$$
\mathbb{E} \sum_{k \geq 1}\left|\left(\hat{X}^{n}(t)-\hat{X}^{m}(t)\right) e_{k}\right|_{H}^{2} \lambda_{k}^{2 \rho} \leq \mathbb{E} \int_{T-\delta}^{T}\left|\hat{Q}^{n}(s)-\hat{Q}^{m}(s)\right|_{\mathcal{K}}^{2} d s
$$

where $\hat{X}^{n}$ and $\hat{X}^{m}$ are defined as in $\frac{\text { ldefx }}{3.17 \text { ) }}$ with $Q_{n}$ replaced by $\hat{Q}^{n}$ and we get, noticing that $\left|\langle X, Z\rangle_{L_{2}(H)}\right| \leq\left(\sum_{k=1}^{\infty}\left|X e_{k}\right|^{2} \lambda_{k}^{2 \rho}\right)^{1 / 2}|Z|_{\mathcal{K}}$

$$
\begin{aligned}
& \mathbb{E} \int_{T-\delta}^{T}\left|\hat{Q}^{n}(s)-\hat{Q}^{m}(s)\right|_{\mathcal{K}}^{2} d s \leq \mathbb{E} \sup _{t \in[T-\delta, T]}\left|\left\langle\hat{X}^{n}(T)-\hat{X}^{m}(T), \hat{P}^{n}(T)-\hat{P}^{m}(T)\right\rangle_{L_{2}(H)}\right| \\
& +\mathbb{E} \int_{T-\delta}^{T}\left|\left\langle J_{n} S(s) J_{n}-J_{m} S(s) J_{m}, \hat{X}^{n}(s)-\hat{X}^{m}(s)\right\rangle_{L_{2}(H)}\right| d s \\
& +\mathbb{E} \int_{T-\delta}^{T}\left|\left\langle C^{\prime}(s)\left[J_{n} P(s) J_{n}-J_{m} P(s) J_{m}\right] C(s), \hat{X}^{n}(s)-\hat{X}^{m}(s)\right\rangle_{L_{2}(H)}\right| d s \\
& +\mathbb{E} \int_{T-\delta}^{T}\left|\left\langle X^{n}(s)-X^{m}(s), C^{\prime}(s)\left(\hat{Q}^{n}(s)-\hat{Q}^{m}(s)\right)\right\rangle_{L_{2}(H)}\right| d s \\
& \left.+\mathbb{E} \int_{T-\delta}^{T}\left|\left\langle X^{n}(s)-X^{m}(s),\left(\hat{Q}^{n}(s)-\hat{Q}^{m}(s)\right) C(s)\right\rangle_{L_{2}(H)}\right| d s\right)=I_{1}+I_{2}+I_{3}+I_{4}+I_{5} .
\end{aligned}
$$

We have

$$
\begin{gathered}
I_{1} \leq \mathbb{E}\left(\sum_{k=1}^{\infty} \lambda_{k}^{2 \rho}\left|\left(\hat{X}^{n}(T)-\hat{X}^{m}(T)\right) e_{k}\right|^{2}\right)^{1 / 2}\left|\hat{P}^{n}(T)-\hat{P}^{m}(T)\right|_{\mathcal{K}} \\
\leq \frac{l}{2} \mathbb{E} \int_{T-\delta}^{T}\left|\hat{Q}^{n}(s)-\hat{Q}^{m}(s)\right|_{\mathcal{K}}^{2} d s+\frac{1}{2 l}\left|\hat{P}^{n}(T)-\hat{P}^{m}(T)\right|_{\mathcal{K}}^{2} \\
I_{2}+I_{3} \leq \frac{l}{2} \mathbb{E} \int_{T-\delta}^{T} \sum_{k=1}^{\infty} \lambda_{k}^{2 \rho}\left|\left(\hat{X}^{n}(s)-\hat{X}^{m}(s)\right) e_{k}\right|^{2} d s+\frac{1}{2 l} \mathbb{E} \int_{T-\delta}^{T} \mid\left\langle J_{n} S(s) J_{n}-\left.J_{m} S(s) J_{m}\right|_{\mathcal{K}} ^{2} d s\right. \\
+\frac{1}{2 l} \mathbb{E} \int_{T-\delta}^{T}\left|C^{\prime}(s)\left[J_{n} P(s) J_{n}-J_{m} P(s) J_{m}\right] C(s)\right|_{\mathcal{K}}^{2} d s,
\end{gathered}
$$




$$
\begin{gathered}
I_{4} \leq \frac{1}{2 l} \mathbb{E} \int_{T-\delta}^{T} \sum_{k=1}^{\infty} \lambda_{k}^{2 \rho}\left|\left(\hat{X}^{n}(s)-\hat{X}^{m}(s)\right) e_{k}\right|^{2} d s+\frac{l}{2} \mathbb{E} \int_{T-\delta}^{T}\left|\hat{Q}^{n}(s)-\hat{Q}^{m}(s)\right|_{\mathcal{K}}^{2} d s \\
\leq \frac{\delta}{2 l} \mathbb{E} \int_{T-\delta}^{T}\left|\hat{Q}^{n}(s)-\hat{Q}^{m}(s)\right|_{\mathcal{K}}^{2} d s+\frac{l}{2} \mathbb{E} \int_{T-\delta}^{T}\left|\hat{Q}^{n}(s)-\hat{Q}^{m}(s)\right|_{\mathcal{K}}^{2} d s
\end{gathered}
$$

$I_{5}$ can be treated as $I_{4}$, following (3.23).

Summarizing and choosing $l$ small enough (depending only on the constants introduced in (2.1), we finally get

$$
\begin{aligned}
& \mathbb{E} \int_{T-\delta}^{T}\left|\hat{Q}^{n}(s)-\hat{Q}^{m}(s)\right|_{\mathcal{K}}^{2} d s \leq C\left(\mathbb{E}\left|\hat{P}^{n}(T)-\hat{P}^{m}(T)\right|_{\mathcal{K}}^{2}+\mathbb{E} \int_{T-\delta}^{T}\left|J_{n} S(s) J_{n}-J_{m} S(s) J_{m}\right|_{\mathcal{K}}^{2} d s\right. \\
& \left.+\mathbb{E} \int_{T-\delta}^{T}\left|C^{\prime}(s) J_{n} P(s) J_{n} C(s)-C^{\prime}(s) J_{m} P(s) J_{m} C(s)\right|_{\mathcal{K}}^{2} d s+\delta \mathbb{E} \int_{T-\delta}^{T}\left|\hat{Q}^{n}(s)-\hat{Q}^{m}(s)\right|_{\mathcal{K}}^{2} d s\right)
\end{aligned}
$$

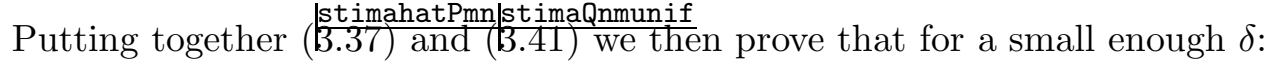

$$
\begin{aligned}
& \lim _{m, n \rightarrow \infty} \mathbb{E} \sup _{t \in[T-\delta, T]}\left|\hat{P}^{n}(t)-\hat{P}^{m}(t)\right|_{\mathcal{K}}^{2}=0 \\
& \lim _{m, n \rightarrow \infty} \mathbb{E} \int_{T-\delta}^{T}\left|\hat{Q}^{n}(s)-\hat{Q}^{m}(s)\right|_{\mathcal{K}}^{2} d s=0 .
\end{aligned}
$$

Therefore there exist the limit $\hat{P} \in L_{\mathcal{P}}^{2}(\Omega ; C([T-\delta, T] ; \mathcal{K}))$ and $\left.\hat{Q} \in L_{\mathcal{P}}^{2}(\Omega \times[T-\delta, T] ; \mathcal{K})\right)$ such that:

$$
\begin{aligned}
& \lim _{n \rightarrow \infty} \mathbb{E} \sup _{t \in[T-\delta, T]}\left|\hat{P}^{n}(t)-\hat{P}(t)\right|_{\mathcal{K}}^{2}=0 \\
& \lim _{m \rightarrow \infty} \mathbb{E} \int_{T-\delta}^{T}\left|\hat{Q}^{n}(s)-\hat{Q}(s)\right|_{\mathcal{K}}^{2} d s=0 .
\end{aligned}
$$

hatPlim

Step 3: construction of $\Gamma$. Being the equation linear, thanks to $\frac{\text { (hatplim }}{(3.42)}$ and $\frac{\text { hatolim }}{(3.43)}$, we obtain the following relation:

$$
\begin{aligned}
\hat{P}(t) & =e^{(T-t) A} M e^{(T-t) A}+\int_{t}^{T} e^{(s-t) A} C^{\prime}(s) P(s) C(s) e^{(s-t) A} d s \\
& +\int_{t}^{T} e^{(s-t) A} S(s) e^{(s-t) A} d s+\int_{t}^{T} e^{(s-t) A}\left(C^{\prime}(s) \hat{Q}(s)+\hat{Q}(s) C(s)\right) e^{(s-t) A} d s \\
& +\int_{t}^{T} e^{(s-t) A} \hat{Q}(s) e^{(s-t) A} d W(s) \quad \mathbb{P}-\text { a.s. }
\end{aligned}
$$

The fact that $\hat{P} \in L_{\mathcal{P}, S}^{2}(\Omega ; C([T-\delta, T] ; L(H)))$ follows from Remark $\frac{\text { stima-a-priori-P-noto }}{3.3}$. So far we have that the map $\Gamma$ such that $\Gamma(P)=\hat{P}$ is actually defined from the space $L_{\mathcal{P}, S}^{2}(\Omega ; C([T-\delta, T] ; L(H))$ into itself.

Step 4: $\Gamma$ is a contraction for a suitable $\delta$. Let $P^{1}$ and $P^{2}$ two elements of $L_{\mathcal{P}, S}^{2}(\Omega ; C([T-$ $\delta, T] ; L(H))$, then we can evaluate the difference between $\Gamma\left(P^{1}\right)$ and $\Gamma\left(P^{2}\right)$. Indeed we have:

$$
\begin{aligned}
\left(\hat{P}^{1}-\hat{P}^{2}\right)(t) & =\int_{t}^{T} e^{(s-t) A} C^{\prime}(s)\left(P^{1}-P^{2}\right)(s) C(s) e^{(s-t) A} d s \\
& +\int_{t}^{T} e^{(s-t) A}\left[C^{\prime}(s)\left(\hat{Q}^{1}-\hat{Q}^{2}\right)(s)+\left(\hat{Q}^{1}-\hat{Q}^{2}\right)(s) C(s)\right] e^{(s-t) A} d s \\
& +\int_{t}^{T} e^{(s-t) A}\left(\hat{Q}^{1}-\hat{Q}^{2}\right)(s) e^{(s-t) A} d W(s) \quad \mathbb{P}-\text { a.s. }
\end{aligned}
$$




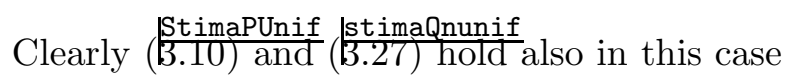

$$
\begin{aligned}
& \mathbb{E} \sup _{u \in[T-\delta, T]}\left|\left(\bar{P}^{1}-\bar{P}^{2}\right)(u)\right|_{L(H)}^{2} \\
& \leq C\left(\delta \mathbb{E} \sup _{u \in[T-\delta, T]}\left|\left(P^{1}-P^{2}\right)(u)\right|_{L(H)}^{2}+\delta^{1-2 \rho}\left(\mathbb{E} \int_{T-\delta}^{T}\left|\left(\hat{Q}^{1}-\hat{Q}^{2}\right)(u)\right|_{\mathcal{K}}^{2} d u\right),\right.
\end{aligned}
$$

with the constant $C$ depending on the constants $M_{C}$ and $T$ but not on $\delta$. And the same holds for $\hat{Q}^{1}-\hat{Q}^{2}$ :

$$
\mathbb{E} \int_{T-\delta}^{T}\left|\left(\hat{Q}^{1}-\hat{Q}^{2}\right)(s)\right|_{\mathcal{K}}^{2} d s \leq C\left(\delta\left|P^{1}-P^{2}\right|_{L_{\mathcal{P}}^{2}(\Omega ; C([T-\delta, T] ; L(H)))}^{2}+\delta^{1-2 \rho} \mathbb{E} \int_{T-\delta}^{T}\left|\left(\hat{Q}^{1}-\hat{Q}^{2}\right)(s)\right|_{\mathcal{K}}^{2} d s\right)
$$

So we can find a $\delta$ small enough such,that $\Gamma$ is a contraction and there's a fixed point $P$. The couple $(P, \hat{Q})$, where $\hat{Q}$ is defined in $(\underline{\mid \mathbf{B} .44} \mid)$ is the mild solution in $[T-\delta, T]$.

Step 5: construction of the mild solution Since the problem is linear and the value of $\delta$ depends only on the constants introduced in $[2.1$, can restart on $[T-2 \delta, T-\delta]$ with final datum $P(T-\delta)$. Proceeding backwards we arrive to cover the whole interval $[0, T]$.

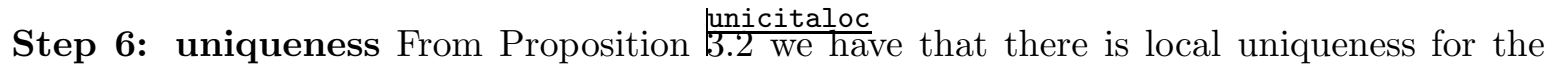
mild solution. Being $\delta_{0}$ independent of the data, we can deduce global uniqueness.

We end the section proving the following stability results for the approximants processes $\hat{P}^{n}$ :

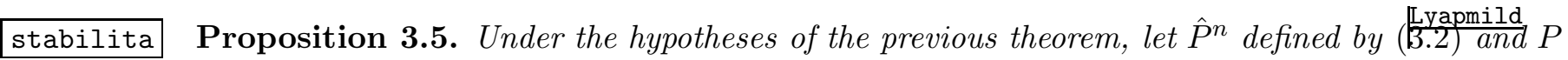
the mild solution just obtained, then the following holds there exists a $\delta>0$ such that for every $\varepsilon<\delta_{1}$ :

$$
\lim _{n \rightarrow \infty} \mathbb{E} \sup _{t \in[T-\delta, T-\varepsilon]}\left|P(t)-\hat{P}^{n}(t)\right|_{L(H)}^{2}=0
$$

Proof. For every $t \in[0, T]$ we have

$P(t)-\hat{P}^{n}(t)=\mathbb{E}^{\mathcal{F}_{t}}\left\{e^{(T-t) A^{\prime}}\left(M-J_{n} M J_{n}\right) e^{(T-t) A}+\int_{t}^{T} e^{(s-t) A^{\prime}}\left(S(s)-J_{n} S(s) J_{n}\right) e^{(s-t) A} d s+\right.$

Lyapmild

$\left.\int_{t}^{T} e^{(s-t) A^{\prime}}\left[C^{\prime}(s)\left(P(s)-J_{n} P(s) J_{n}\right) C(s)+C^{\prime}(s)\left(Q(s)-\hat{Q}^{n}(s)\right)+\left(Q(s)-\hat{Q}^{n}(s)\right) C(s)\right] e^{(s-t) A} d s\right\}$, 
thus, assume that $\delta<1$

$$
\begin{aligned}
& \mathbb{E} \sup _{t \in[T-\delta, T-\varepsilon]}\left|\mathbb{E}^{\mathcal{F}_{t}} e^{(T-t) A}\left(M-J_{n} M J_{n}\right) e^{(T-t) A}\right|_{L(H)}^{2} \\
& =\mathbb{E} \sup _{t \in[T-\delta, T-\varepsilon]}\left|\mathbb{E}^{\mathcal{F}_{t}} e^{(T-\varepsilon-t) A} e^{\varepsilon A}\left(M-J_{n} M J_{n}\right) e^{\varepsilon A} e^{(T-\varepsilon-t) A}\right|_{L(H)}^{2} \\
& \leq 4 \varepsilon^{-2 \rho} \mathbb{E}\left|M-J_{n} M J_{n}\right|_{\mathcal{K}}^{2}, \\
& \mathbb{E} \sup _{t \in[T-\delta, T-\varepsilon]}\left|\mathbb{E}^{\mathcal{F}_{t}} \int_{t}^{T} e^{(s-t) A} C^{\prime}(s)\left(P(s)-J_{n} P(s) J_{n}\right) C(s) e^{(s-t) A} d s\right|_{L(H)}^{2} \\
& \leq 4 \delta^{1-2 \rho} \mathbb{E} \int_{T-\delta}^{T}\left|C^{\prime}(s)\left(P(s)-J_{n} P(s) J_{n}\right) C(s)\right|_{\mathcal{K}}^{2} d s, \\
& \mathbb{E} \sup _{t \in[T-\delta, T-\varepsilon]}\left|\mathbb{E}^{\mathcal{F}_{t}} \int_{t}^{T} e^{(s-t) A}\left[C^{\prime}(s)\left(Q(s)-\hat{Q}^{n}(s)\right)+\left(Q(s)-\hat{Q}^{n}(s)\right) C(s)\right] e^{(s-t) A} d s\right|_{L(H)}^{2} \\
& \leq 2 \sup _{t \in[T-\delta, T]}\left(\mathbb { E } ^ { \mathcal { F } _ { t } } \int _ { t } ^ { T } \frac { M _ { C } } { ( s - t ) ^ { \rho } } | ( Q ( s ) - \overline { Q } ^ { n } ( s ) | _ { \mathcal { K } } d s ) ^ { 2 } \leq 8 M _ { C } ^ { 2 } \delta ^ { 1 - 2 \rho } \mathbb { E } \int _ { T - \delta } ^ { T } | \left(Q(s)-\left.\bar{Q}^{n}(s)\right|_{\mathcal{K}} ^{2} d s,\right.\right. \\
& \mathbb{E} \sup _{t \in[T-\delta, T]}\left|\int_{t}^{T} e^{(s-t) A}\left(J_{n} S(s) J_{n}-S(s)\right) e^{(s-t) A} d s\right|_{L(H)}^{2} \leq \delta^{1-2 \rho} \mathbb{E} \int_{r}^{T}\left|S(s)-J_{n} S(s) J_{n}\right|_{\mathcal{K}}^{2} d s .
\end{aligned}
$$

Summing up all these estimates we deduce that there exists a constant $C$ depending only on $M_{C}, \rho$ such that:

$$
\begin{aligned}
& \mathbb{E} \sup _{t \in[T-\delta, T-\varepsilon]}\left|P(t)-\hat{P}^{n}(t)\right|_{L(H)}^{2} \leq C\left(\varepsilon^{-2 \rho} \mathbb{E}\left|M-J_{n} M J_{n}\right|_{\mathcal{K}}^{2}+\delta^{1-2 \rho} \mathbb{E} \int_{T-\delta}^{T}\left|P(s)-J_{n} P(s) J_{n}\right|_{\mathcal{K}}^{2} d s\right. \\
& \left.\delta^{1-2 \rho} \mathbb{E} \int_{T-\delta}^{T}\left|Q(s)-\hat{Q}^{n}(s)\right|_{\mathcal{K}}^{2} d s+\delta^{1-2 \rho} \mathbb{E} \int_{r}^{T}\left|S(s)-J_{n} S(s) J_{n}\right|_{\mathcal{K}}^{2} d s\right) .
\end{aligned}
$$

Thanks to previous considerations in particular $\frac{(\text { hatol } 13 \text { im }}{3.43}$, and recalling that by dominated convergence theorem $\mathbb{E} \int_{T-\delta}^{T}\left|P(s)-J_{n} P(s) J_{n}\right|_{\mathcal{K}}^{2} \rightarrow 0$, we deduce the thesis.

\section{Backward Stochastic Riccati Equations and LQ Optimal Control}

Besides hypotheses $\frac{\text { annyp }}{2.11}$ let us fix $T>S>0$ and consider the following infinite dimensional stochastic control problem, with state equation given by

$$
\left\{\begin{array}{l}
d y(t)=(A y(t)+B(t) u(t)) d t+C(t) y(t) d W(t) \quad S \leq r \leq t \leq T \\
y(r)=x
\end{array}\right.
$$

where $u$ is the control and $_{\text {foenhyp }}$ takes values in another Hilbert space $U$.

hypB Besides hypothesis 2.1 we assume that

A4) We assume that $B \in L_{\mathcal{P}, S}^{\infty}(\Omega \times[0, T] ; L(U ; H))$. We denote with $M_{B}$ a positive constant such that:

$$
|B(t, \omega)|_{L(U ; H)}<M_{B}, \quad \mathbb{P}-\text { a.s. and for a.e. } t \in(0, T) .
$$

We recall the definition of mild solution.

ef-mild-stato Definition 4.1. Given $x \in H$ and $u \in L_{\mathcal{P}}^{2}(\Omega \times[t, T] ; U)$, a mild solution of (Istato 1 is a process $y \in L_{\mathcal{P}}^{2}(\Omega \times[t, T] ; H)$ such that, almost everywhere in $\Omega \times[t, T]$,

$$
y(s)=e^{(s-t) A} x+\int_{t}^{s} e^{(s-\sigma) A} B(\sigma) u(\sigma) d \sigma+\int_{t}^{s} e^{(s-\sigma) A} C(\sigma) y(\sigma) d W(\sigma) .
$$

The following existence and uniqueness result holds: 


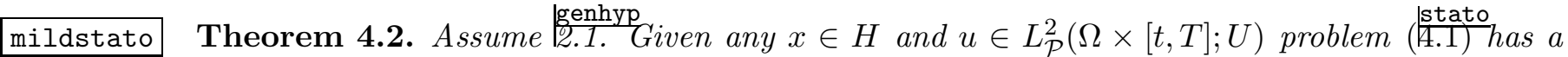
unique mild solution $y \in C_{\mathcal{P}}\left([t, T] ; L^{2}(\Omega ; H)\right)$. Moreover,

$$
\sup _{s \in[t, T]} \mathbb{E}|y(s)|^{2} \leq C_{2}\left[|x|^{2}+\mathbb{E} \int_{t}^{T}|u(s)|^{2} d s\right]
$$

for a suitable constant $C_{2}$ depending only on $T, M_{B}, M_{C}$ (notice that $C_{2} \geq 1$ ).

Finally if $p>2$ and

$$
\mathbb{E}\left(\int_{t}^{T}|u(s)|^{2} d s\right)^{\frac{p}{2}}<\infty,
$$

then we have that $y \in L_{\mathcal{P}}^{p}(\Omega ; C([t, T] ; H))$ and

$$
\mathbb{E} \sup _{s \in[t, T]}|y(s)|^{p} \leq C_{p}\left[|x|^{p}+\mathbb{E}\left(\int_{t}^{T}|u(s)|^{2} d s\right)^{\frac{p}{2}}\right]
$$

for some positive constant $C_{p}$ depending on $p, T, M_{B}, M_{C}$.

The cost functional to minimize over all processes taking values in $L_{\mathcal{P}}^{2}(\Omega \times[0, T], U)$ - the space of admissible controls is

$$
\mathbb{E} \int_{0}^{T}\left(|\sqrt{S}(s) y(s)|_{H}^{2}+|u(s)|_{H}^{2}\right) d s+\mathbb{E}\langle M y(T), y(T)\rangle_{H}
$$

Associated to this Linear and Quadratic control problem we have the following Backward Stochastic Riccati Equation (BSRE), see [2, 13] and 7 f for the present infinite dimensional version:

$$
\left\{\begin{array}{rl}
-d P(t)= & \left(A P(t)+P(t) A+C^{\prime}(t) P(t) C(t)+C^{\prime}(t) Q(t)+Q(t) C(t)\right) d t \\
& -\left(P(t) B(t) B^{*}(t) P(t)-S(t)\right) d t+Q(t) d W(t) \\
P(T)=M & t \in[0, T]
\end{array}\right.
$$

In this section we will prove that such equation has a unique mild solution, in the sense of definition 3.1 improving the result obtained in 17. . To be more specific we have

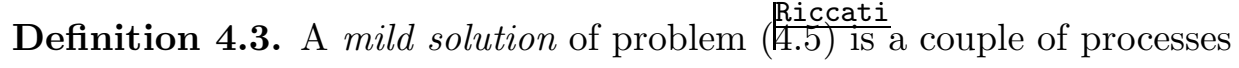

$$
(P, Q) \in L_{\mathcal{P}, S}^{2}(\Omega, C([0, T] ; \Sigma(H))) \times L_{\mathcal{P}}^{2}\left(\Omega \times[0, T] ; \mathcal{K}_{s}\right)
$$

that solves the following equation, for all $t \in[0, T]$ :

$$
\begin{aligned}
P(t) & =e^{(T-t) A^{\prime}} M e^{(T-t) A}+\int_{t}^{T} e^{(s-t) A^{\prime}} S(s) e^{(s-t) A} d s \\
& \left.+\int_{t}^{T} e^{(s-t) A^{\prime}}\left[C^{\prime}(s) P(s) C(s)-P\right)(s) B(s) B^{\prime}(S) P(s)+C^{\prime}(s) Q(s)+Q(s) C(s)\right] e^{(s-t) A} d s \\
& +\int_{t}^{T} e^{(s-t) A^{*}} Q(s) e^{(s-t) A} d W(s) \quad \mathbb{P} \text { - a.s. }
\end{aligned}
$$

We have indeed:

main Theorem 4.4. Assume that hypotheses $\frac{\text { enpyp }}{2.1}$ and $\mathrm{A}_{4}$ ) hold true. Then there exists a unique mild solution $(P, Q)$ of equation (4.J) in $[0, T]$. Moreover $P \in L_{\mathcal{P}, S}^{\infty}\left(\Omega \times(0, T) ; \Sigma^{+}(H)\right)$. Moreover, fix $T>0$ and $x \in H$, then

1. there exists a unique control $\bar{u} \in L_{\mathcal{P}}^{2}(\Omega \times[0, T] ; U)$ such that

$$
J(0, x, \bar{u})=\inf _{u \in L_{\mathcal{P}}^{2}(\Omega \times[0, T] ; U)} J(0, x, u) ;
$$


2. if $\bar{y}$ is the mild solution of the state equation corresponding to $\bar{u}$ (that is, the optimal state), then $\bar{y}$ is the unique mild solution to the closed loop equation

$$
\left\{\begin{array}{l}
d \bar{y}(r)=\left[A \bar{y}(r)-B(r) B^{\prime}(r) P(r) \bar{y}(r)\right] d r+C \bar{y}(r) d W(r), \\
\bar{y}(0)=x
\end{array}\right.
$$

3. the following feedback law holds $\mathbb{P}$-a.s. for almost every s:

$$
\bar{u}(s)=-B^{\prime}(s) P(s) \bar{y}(s)
$$

4. the optimal cost is given by $J(0, x, \bar{u})=\langle P(0) x, x\rangle_{H}$.

Before going into the details of the proof, we establish the following-priori estimate.

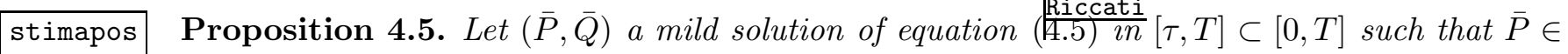
$L_{\mathcal{P}, S}^{\infty}(\Omega \times[\tau, T], \Sigma(H))$, then the following holds for every $t \in[\tau, T]$ :

(i) for all $t \in[\tau, T], \bar{P}(t) \in \Sigma^{+}(H), \quad \mathbb{P}-$ a.s..

(ii) for all $t \in[\tau, T]$,

$$
\begin{aligned}
& |\bar{P}(t)|_{L(H)} \leq C_{2}\left(|M|_{L_{\mathcal{P}, S}^{\infty}\left(\Omega, \mathcal{F}_{T} ; L(H)\right)}+(T-\tau)|S|_{L_{\mathcal{P}, S}^{\infty}(\Omega \times[\tau, T], L(H))}\right) \quad \mathbb{P}-\text { a.s. } \\
& \text { where } C_{2} \text { is given in (1stima- }
\end{aligned}
$$

Proof. Step 1 [Fundamental relation for the Lyapunov equation]. Let $(P, Q)$ be the unique mild solution to the Lyapunov equation (3.2) and let $y^{t, x}$ be the mild solution to (15.1), we claim that for all $t \in[0, T], x \in H$, it holds,

$$
\begin{aligned}
\langle P(t) x, x\rangle_{H} & =\mathbb{E}^{\mathcal{F}_{t}}\left\langle M y^{t, x}(T), y^{t, x}(T)\right\rangle+\mathbb{E}^{\mathcal{F}_{t}} \int_{t}^{T}\left\langle S(s) y^{t, x}(s), y^{t, x}(s)\right\rangle_{H} d s \\
& -2 \mathbb{E}^{\mathcal{F}_{t}} \int_{t}^{T}\left\langle P(s) B^{\prime}(s) y^{t, x}(s), u(s)\right\rangle d s, \quad \mathbb{P} \text {-a.s.. }
\end{aligned}
$$

Let us prove the claim. We will use again the approximants processes $\left(\hat{P}^{n}, \hat{Q}^{n}\right)$ introduced in the proof of theorem 3.4 . From proposition 3.5 we know that there's a $\delta$ small enough such that for every $\varepsilon<\delta$ :

$$
\lim _{n \rightarrow \infty} \mathbb{E} \sup _{t \in[T-\delta, T-\varepsilon]}\left|P(t)-\hat{P}^{n}(t)\right|_{L(H)}^{2}=0 .
$$

On the other hand we have already notjced that $\left(\hat{P}^{n}, \hat{Q}^{n}\right)$ is a solution in the sense of Proposition 2.1 of [z], therefore by Theorem 5.6 of [t] we have that: for all $t \in[0, T], x \in H$, it holds, $\mathbb{P}$-a.s., that

$$
\begin{aligned}
\left\langle\hat{P}^{n}(t) x, x\right\rangle_{H} & =\mathbb{E}^{\mathcal{F}_{t}}\left\langle\hat{P}^{n}(T-\varepsilon) y^{t, x}(T-\varepsilon), y^{t, x}(T-\varepsilon)\right\rangle_{H}+\mathbb{E}^{\mathcal{F}_{t}} \int_{t}^{T-\varepsilon}\left\langle S(s) y^{t, x, u}(s), y^{t, x, u}(s)\right\rangle_{H} d s \\
& +\mathbb{E}^{\mathcal{F}_{t}} \int_{t}^{T-\varepsilon}\left\langle\left[C^{\prime}(s) \hat{P}^{n}(s) C(s)-C^{\prime}(s) J_{n} P(s) J_{n} C(s)\right] y^{t, x, u}(s), y^{t, x, u}(s)\right\rangle_{H} d s \\
& -2 \mathbb{E}^{\mathcal{F}_{t}} \int_{t}^{T-\varepsilon}\left\langle\hat{P}^{n}(s) B^{\prime}(s) y^{t, x, u}(s), u(s)\right\rangle_{H} d s
\end{aligned}
$$

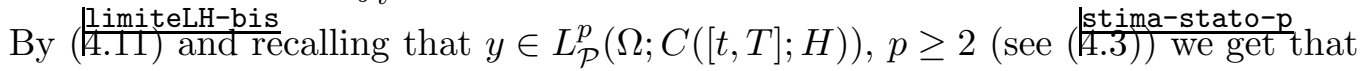

$$
\int_{t}^{T-\varepsilon}\left\langle\hat{P}^{n}(s) C(s) y^{t, x, u}(s), C(s) y^{t, x, u}(s)\right\rangle d s \rightarrow \int_{t}^{T-\varepsilon}\left\langle P(s) C(s) y^{t, x, u}(s), C(s) y^{t, x, u}(s)\right\rangle d s
$$

in $L^{1}$ norm. Moreover, since $\mathbb{E} \sup _{t \in[0, T]}|P(t)|_{L(H)}^{2}<+\infty$, by Dominated convergence theorem we obtain that

$$
\int_{t}^{T-\varepsilon}\left\langle P(s) J_{n} C(s) y^{t, x, u}(s), J_{n} C(s) y^{t, x, u}(s)\right\rangle d s \rightarrow \int_{t}^{T-\varepsilon}\left\langle P(s) C(s) y^{t, x, u}(s), C(s) y^{t, x, u}(s)\right\rangle d s
$$


again in $L^{1}$ norm.

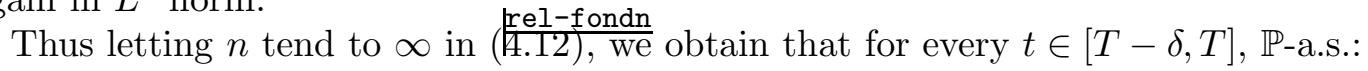

$$
\begin{aligned}
\langle P(t) x, x\rangle_{H} & =\mathbb{E}^{\mathcal{F}_{t}}\left\langle P(T-\varepsilon) y^{t, x}(T-\varepsilon), y^{t, x}(T-\varepsilon)\right\rangle_{H}+\mathbb{E}^{\mathcal{F}_{t}} \int_{t}^{T-\varepsilon}\left\langle S(s) y^{t, x, u}(s), y^{t, x, u}(s)\right\rangle d s \\
& -2 \mathbb{E}^{\mathcal{F}_{t}} \int_{t}^{T-\varepsilon}\left\langle P(s) B^{\prime}(s) y^{t, x, u}(s), u(s)\right\rangle d s
\end{aligned}
$$

Now, thanks again to $\mathbb{E} \sup _{t \in[0, T]}|P(t)|_{L(H)}^{2}<+\infty$, we can let $\varepsilon$ going to 0 and get that for every $x \in H$, and every $t \in[T-\delta, T], \mathbb{P}$-a.s.:

$$
\begin{array}{r}
\langle P(t) x, x\rangle_{H}=\mathbb{E}^{\mathcal{F}_{t}}\left\langle M y^{t, x}(T), y^{t, x}(T)\right\rangle_{H}+\mathbb{E}^{\mathcal{F}_{t}} \int_{t}^{T}\left\langle S(s) y^{t, x, u}(s), y^{t, x, u}(s)\right\rangle d s \\
-2 \mathbb{E}^{\mathcal{F}_{t}} \int_{t}^{T}\left\langle P(s) B^{\prime}(s) y^{t, x, u}(s), u(s)\right\rangle d s
\end{array}
$$

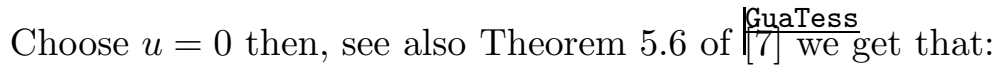

$$
\sup _{x \in H,|x|_{H}=1}\left|\langle P(t) x, x\rangle_{H}\right| \leq C_{2}\left(|M|_{L_{S}^{\infty}\left(\Omega, \mathcal{F}_{T}, P\right)}+T|S|_{L_{\mathcal{P}, S}^{\infty}(\Omega \times(0, T) ; L(H))}\right), \quad \forall t \in[T-\delta, T] .
$$

We can prove relation $\left(\frac{\text { rel-filis }}{1.13}\right.$ ) on the interval $[T-2 \delta, T-\delta]$ (notice that $\delta$ does not depend on $M$ ) and so on to cover the whple interval $[0, T]$, because $P(T-k \delta) \in L(H)$ for every $k=0,1,2,3, \ldots$ and thus we can extend (15.15) to the whole $[0, T]$.

Step 2: upper bound Let $(\bar{P}, \bar{Q})$ be the mild solution of the BSRE $\frac{\text { Riccati }}{\left(\frac{1}{1.5}\right) \text { in }}[\tau, T]$, we can see such couple of processes as the mild solution to the following Lyapunov equation, for $t \in[\tau, T]$ :

$$
\left\{\begin{array}{l}
-d \bar{P}(t)=\left(A \bar{P}(t)+\bar{P}(t) A+C^{\prime}(t) \bar{P}(t) C(t)+C^{\prime}(t) \bar{Q}(t)+\bar{Q}(t) C(t)+\bar{S}(t)\right) d t+\bar{Q}(t) d W(t), \\
\bar{P}(T)=M
\end{array}\right.
$$

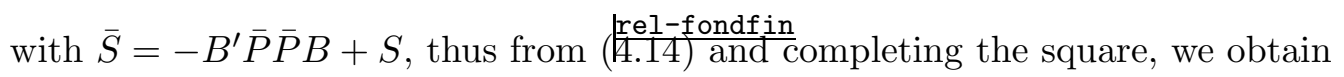

$$
\begin{aligned}
\langle\bar{P}(t) x, x\rangle_{H} & =\mathbb{E}^{\mathcal{F}_{t}}\left\langle M y^{t, x}(T), y^{t, x}(T)\right\rangle_{H}+\mathbb{E}^{\mathcal{F}_{t}} \int_{t}^{T}|u(s)|^{2} d s \\
& +\mathbb{E}^{\mathcal{F}_{t}} \int_{t}^{T}\left\langle S(s) y^{t, x, u}(s), y^{t, x, u}(s)\right\rangle d s-\mathbb{E}^{\mathcal{F}_{t}} \int_{t}^{T}\left|\bar{P}(s) B^{\prime}(s) y^{t, x, u}(s)+u(s)\right|^{2} d s
\end{aligned}
$$

So, choosing the admissible control $u=0$, we get:

$$
\begin{aligned}
\langle\bar{P}(t) x, x\rangle_{H} & =\mathbb{E}^{\mathcal{F}_{t}}\left\langle M y^{t, x, 0}(T), y^{t, x, 0}(T)\right\rangle_{H}+\mathbb{E}^{\mathcal{F}_{t}} \int_{t}^{T}\left\langle S(s) y^{t, x, 0}(s), y^{t, x, 0}(s)\right\rangle d s \\
& -\mathbb{E}^{\mathcal{F}_{t}} \int_{t}^{T}\left|\bar{P}(s) B^{\prime}(s) y^{t, x, 0}(s)\right|^{2} d s
\end{aligned}
$$

From which we deduce the following upper bound

$$
\langle\bar{P}(t) x, x\rangle_{H} \leq C_{2}\left(|M|_{L_{S}^{\infty}\left(\Omega, \mathcal{F}_{T}, P\right)}+T|S|_{L_{\mathcal{P}, S}^{\infty}(\Omega \times(0, T) ; L(H))}\right), \quad \forall t \in[\tau, T] .
$$

Step 3: lower bound Let us consider the following equation for initial time $t \in[\tau, T]$ and initial state $x$ :

$$
\left\{\begin{array}{l}
d \bar{y}(s)=\left[A \bar{y}(s)-B(s) B^{\prime}(s) \bar{P}(s) \bar{y}(s)\right] d s+C \bar{y}(s) d W(s), \quad s \in[t, T] \\
\bar{y}(t)=x
\end{array}\right.
$$




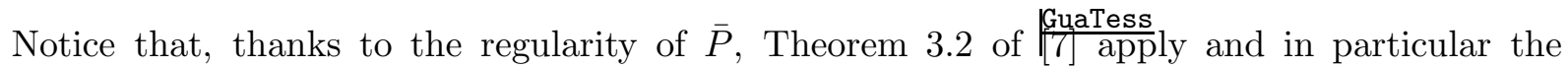
following estimates holds true for the solution $\bar{y}^{t, x}$, for every $t \in[\tau, T]$ :

$$
\mathbb{E}^{\mathcal{F}_{t}} \sup _{s \in[t, T]}|\bar{y}(s)|^{p} \leq C_{p}|x|^{p}, \quad \forall p \geq 2
$$

where $C_{p}$ depends also on the $L^{\infty}$ norm of $\bar{P}_{\text {refondRiccati }}$ There $\bar{u}(s)=B^{\prime}(s) \bar{P}(s) \bar{y}^{t, x}(s)$ is an admissible control, i.e. $\bar{u} \in L_{\mathcal{P}}^{2}(\Omega \times[t, T], U)$, and (17) corresponds to

$$
\langle\bar{P}(t) x, x\rangle_{H}=\mathbb{E}^{\mathcal{F}_{t}}\left[\left\langleM \overline { y } ^ { t , x } \left((T), \bar{y}^{t, x}((T)\rangle_{H}+\int_{t}^{T}\left(\mid B^{\prime}(s) \bar{P}(s) \bar{y}^{t, x}\left(\left.(s)\right|^{2}+\left|\sqrt{S(s)} \bar{y}^{t, x}(s)\right|^{2}\right) d s\right], \mathbb{P}-a . s .\right.\right.\right.
$$

relfondR

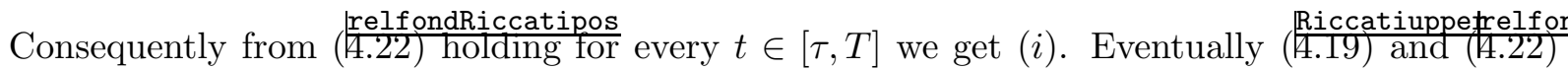
imply $(i i)$.

We are now in the position to prove Theorem main

\section{Proof of Theorem 4.4 .}

\section{Step 1: local existence and uniqueness}

In order to be able to follow the same argument not only on $[T-\delta, T]$ but also on $[T-2 \delta, T-\delta]$ and so on (with the same $\delta$ ) we prove existence of a solution (for notational convenience on $[T-\delta, T])$ with generic final condition $\tilde{M} \in L_{\mathcal{P}, S}^{\infty}\left(\Omega, \mathcal{F}_{T} ; L(H)\right)$ with

$$
|\widetilde{M}|_{L_{\mathcal{P}, S}^{\infty}\left(\Omega, \mathcal{F}_{T} ; L(H)\right)}<C_{2}\left(|M|_{L_{\mathcal{P}, S}^{\infty}\left(\Omega, \mathcal{F}_{T} ; L(H)\right)}+T|S|_{L_{\mathcal{P}, S}^{\infty}(\Omega \times[0, T], L(H))}\right)
$$

We fix a number $r$ with

$$
r>C_{2}^{2}|M|_{L_{S}^{\infty}\left(\Omega, \mathcal{F}_{T}, P\right)}+2 C_{2}^{2} T|S|_{L_{\mathcal{P}, S}^{\infty}(\Omega \times(0, T) ; L(H))}
$$

where $C_{2}$ is the the constant obtained in Proposition

$$
\mathcal{B}(r)=\left\{P \in L_{\mathcal{P}, S}^{2}(\Omega ; C([T-\delta, T] ; L(H))): \sup _{t \in[T-\delta, T]}|P(t, \omega)|_{L(H)} \leq r \quad \mathbb{P} \text {-a.s. }\right\}
$$

where $\delta>0$ will be fixed later on. On $\mathcal{B}(r)$ we construct the map $\Lambda: \mathcal{B}(r) \rightarrow \mathcal{B}(r)$, letting $\Lambda(K)=P$, where $(P, Q)$ is the unique mild solution to $($ B.2. $S-K B B^{*} K$ and $M$ by $\widetilde{M}$ that is verifies

$$
\begin{aligned}
P(t)= & e^{(T-t) A} \widetilde{M} e^{(T-t) A}+\int_{t}^{T} e^{(s-t) A}\left[C^{\prime}(s) P(s) C(s)+C^{\prime}(s) Q(s)+Q(s) C(s)\right] e^{(s-t) A} d s \\
& +\int_{t}^{T} e^{(s-t) A} S(s) e^{(s-t) A} d s+\int_{t}^{T} e^{(s-t) A} K(s) B(s) B^{\prime}(s) K(s) e^{(s-t) A} d s \\
& +\int_{t}^{T} e^{(s-t) A} Q(s) e^{(s-t) A} d W(s)
\end{aligned}
$$

First of all we check that it maps $\mathcal{B}(r)$ into itself. It is enough to show that for all $t \in[T-\delta, T]$ it holds $|\Lambda(K)(t)|_{L(H)} \leq r \mathbb{P}$-a.s. Thanks to (10.9) we have that $\mathbb{P}$-a.s.

$$
\begin{aligned}
& |\Lambda(K)(t)|_{L(H)} \leq C_{2}\left[|\widetilde{M}|_{L_{S}^{\infty}\left(\Omega, \mathcal{F}_{T} ; L(H)\right)}+\delta\left|K B B^{\prime} K\right|_{L_{\mathcal{P}, S}^{\infty}(\Omega \times[T-\delta, T] ; L(H))}\right. \\
& \left.\left.+\delta|S|_{L_{\mathcal{P}, S}^{\infty}(\Omega \times[T-\delta, T] ; L(H))}\right) d s\right] \leq \\
& \leq C_{2}^{2}|M|_{L^{\infty}}+C_{2} r^{2} \delta M_{B}^{2}+2 C_{2}^{2} T|S|_{L_{\mathcal{P}, S}^{\infty}(\Omega \times[0, T] ; L(H))}<r
\end{aligned}
$$


as soon as we choose

$$
\delta<\frac{r-\left(C_{2}^{2}|M|_{L^{\infty}}+2 C_{2}^{2} T|S|_{L_{\mathcal{P}, S}^{\infty}(\Omega \times[0, T] ; L(H))}\right)}{C_{2}^{2} M_{B}^{2} r^{2}} .
$$

Let $K_{1}$ and $K_{2}$ in $B(r)$, then by

$$
\begin{aligned}
\left\langle\left(P^{1}(t)-P^{2}(t)\right) x, x\right\rangle_{H} & =\mathbb{E}^{\mathcal{F}_{t}} \int_{t}^{T}\left\langle K^{1}(s) B(s) B^{\prime}(s)\left(K^{1}(s)-K^{2}(s)\right) y^{t, x, 0}(s), y^{t, x, 0}(s)\right\rangle d s \\
& -\mathbb{E}^{\mathcal{F}_{t}} \int_{t}^{T}\left\langle K^{2}(s) B(s) B^{\prime}(s)\left(K^{1}(s)-K^{2}(s)\right) y^{t, x, 0}(s), y^{t, x, 0}(s)\right\rangle d s,
\end{aligned}
$$

thus, by Hölder inequality,

$$
\begin{aligned}
& \left|\left\langle\left(P^{1}(t)-P^{2}(t)\right) x, x\right\rangle_{H}\right| \leq 2 \mathbb{E}^{\mathcal{F}_{t}} \int_{t}^{T} r M_{B}^{2}\left|K^{1}(s)-K^{2}(s)\right|_{L(H)}\left|y^{t, x, 0}(s)\right|^{2} d s \\
& \leq 2 r M_{B}^{2} \int_{t}^{T}\left(\mathbb{E}^{\mathcal{F}_{t}}\left|K^{1}(s)-K^{2}(s)\right|_{L(H)}^{2}\right)^{1 / 2}\left(\mathbb{E}^{\mathcal{F}_{t}}\left|y^{t, x, 0}(s)\right|^{4}\right)^{1 / 2} d s \\
& \leq 2 r M_{B}^{2} \delta^{2}\left(\sup _{t \in[T-\delta, T]} \mathbb{E}^{\mathcal{F}_{t}}\left|K^{1}(s)-K^{2}(s)\right|_{L(H)}^{2}\right)^{1 / 2}\left(\sup _{t \in[T-\delta, T]} \mathbb{E}^{\mathcal{F}_{t}}\left|y^{t, x, 0}(s)\right|^{4}\right)^{1 / 2}
\end{aligned}
$$

using again Doob inequality and (1stimastatoloop

$$
\mathbb{E} \sup _{t \in[T-\delta, T]}\left|P^{1}(t)-P^{2}(t)\right|_{L(H)}^{2} \leq 16 r^{2} M_{B}^{4} \delta^{4} C_{4} \mathbb{E} \sup _{t \in[T-\delta, T]}\left|K^{1}(t)-K^{2}(t)\right|_{L(H)}^{2}
$$

where $C_{4}=C_{4}(r)$ is given in $\frac{\text { stimastatoloop }}{(1.211)}$. Therefore reducing if necessary the value of $\delta$, we obtain that $\Lambda$ is a contraction.

Step 2: global existence and uniqueness We notice that the choice of $\delta$ depends only on $r$ and the constants introduced in hypotheses to cover the whole interval $[0, T]$.

Final step: synthesis of the optimal control. So far we have proyed the existence and uniqueness of the mild solution for the BSRE, and thanks to Proposition the also have that the first component of the solution $P \in L_{\mathcal{P}, S}^{\infty}(\Omega \times[0, T] ; L(H))$. Consequently the closed loop equation (1.7. is well posed and the associated feedback control is admissible, hence the rest of the claims of the Theorem easily follow.

\section{The Lyapunov Equation of the Maximum Principle}

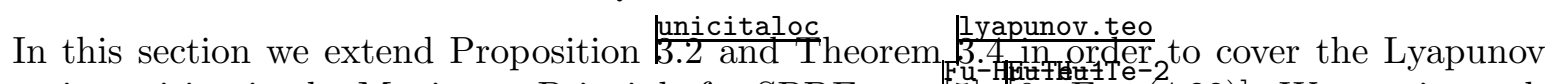
equation arising in the Maximum Principle for SPDE, see [5], [6, Eq. (4.22)]. We rewrite such equation with our notation

$$
\left\{\begin{array}{c}
-d P(t)=-Q(t) d W(t)+\left[A^{*} P(t)+P(t) A+A_{\sharp}^{*}(t) P(t)+P(t) A_{\sharp}^{*}\right] d t \\
+[C(t) P(t) C(t)+C(t) Q(t)+Q(t) C(t)+S(t)] d t \\
P(T)=M,
\end{array}\right.
$$

where $A_{\sharp} \in L_{\mathcal{P}, S}^{\infty}((0, T) \times \Omega ; L(H))$.

The presence of the bounded term $A_{\sharp}$ is completely irrelevant and we will not consider it in the following.

On the contrary it is not possible, in this context, to require Assumption A3). Indeed Assumption A3) has to be replaced by the weaker one

Hypothesis 5.1. A3') $\left.S \in L_{\mathcal{P}, S}^{2}((0, T) \times \Omega ; \mathcal{K})\right)$ and $M \in L_{S}^{\infty}\left(\Omega, \mathcal{F}_{T} ; L(H)\right)$.

(notice that the assumption on $M$ remains unchanged)

Under A3') the statement of the a-priori estimate in Proposition unicitaloc 
Proposition 5.2. Let $(P, Q)$ a mild solution to (BSDEoperatorvalued . Then there exists a $\delta_{0}>0$ just depending on $T$ and the constants $M_{A}, M_{C}$ and $\rho$ introduced in A1) - A2) such that for every $0 \leq \delta \leq \delta_{0}$ the following holds:

$$
|P|_{L^{2}(\Omega ; C([T-\delta, T] ; L(H)))}^{2}+\mathbb{E} \int_{T-\delta}^{T}|Q(s)|_{\mathcal{K}}^{2} d s \leq c\left(\mathbb{E}|M|_{L(H)}^{2}+\delta^{1-2 \rho} \mathbb{E} \int_{T-\delta}^{T}|S(s)|_{\mathcal{K}}^{2} d s\right) .
$$

where $c$ is a positive constant depending on $\delta_{0}, M_{A}, M_{C}, \rho$ and $T$.

Proof. Let us reestimate (stimaS

$$
\begin{aligned}
\mathbb{E} \sup _{t \in[r, T]}\left|\int_{t}^{T} e^{(s-t) A} S(s) e^{(s-t) A} d s\right|_{L(H)}^{2} & \leq \mathbb{E} \sup _{t \in[r, T]}\left(\int_{t}^{T}(s-t)^{-2 \rho} d s \int_{t}^{T}|S(s)|_{\mathcal{K}}^{2} d s\right) \\
& \leq(T-r)^{1-2 \rho} \int_{r}^{T}|S(s)|_{\mathcal{K}}^{2} d s \quad \forall r \in[T-\delta, T]
\end{aligned}
$$

Therefore $\frac{\text { IStimaPUnif_zero }}{(3.9) \text { becomes }}$

$$
\begin{aligned}
& \mathbb{E} \sup _{u \in[T-\delta, T]}|P(u)|_{L(H)}^{2} \\
& \leq C\left(|M|_{L(H)}^{2}+\delta^{2} \mathbb{E} \sup _{u \in[T-\delta, T]}|P(u)|_{L(H)}^{2}+\delta^{1-2} \mathbb{E}_{T} \int_{T-\delta}^{T}|Q(s)|_{\mathcal{K}}^{2} d s+\delta^{1-2 \rho} \mathbb{E} \int_{T-\delta}^{T}|S(s)|_{\mathcal{K}}^{2} d s\right)
\end{aligned}
$$

From which we deduce:

$$
\mathbb{E} \sup _{u \in[T-\delta, T]}|P(u)|_{L(H)}^{2} \leq C\left(|M|_{L(H)}^{2}+\delta^{1-2 \rho} \mathbb{E} \int_{T-\delta}^{T}|Q(s)|_{\mathcal{K}}^{2} d s+\delta^{1-2 \rho} \mathbb{E} \int_{T-\delta}^{T}|S(s)|_{\mathcal{K}}^{2} d s\right)
$$

Regarding the duality argument used to estimate $\mathbb{E} \int_{T-\delta}^{T}|Q(s)|_{\mathcal{K}}^{2} d s$ the only thing to check is that (3.26) still holds:

$$
\begin{aligned}
& \left|\mathbb{E} \int_{T-\delta}^{T}\left\langle X^{n}(s), J_{n} S(s) J_{n}\right\rangle_{L_{2}(H)} d s\right|=\left|\mathbb{E} \int_{T-\delta}^{T} \sum_{k \geq 1}\left\langle X^{n}(s) e_{k}, J_{n} S(s) J_{n} e_{k}\right\rangle_{H} d s\right| \\
& \leq\left(\int_{T-\delta}^{T} \mathbb{E} \sum_{k \geq 1} \lambda_{k}^{2 \rho}\left|X^{n}(s) e_{k}\right|^{2} d s\right)^{1 / 2}\left(\int_{T-\delta}^{T} \mathbb{E} \sum_{k \geq 1} \lambda_{k}^{-2 \rho}\left|J_{n} S(s) J_{n} e_{k}\right|^{2} d s\right)^{1 / 2} \\
& \leq\left(\delta \int_{T-\delta}^{T}\left|Q^{n}(s)\right|_{\mathcal{K}}^{2} d s\right)^{1 / 2}\left(\int_{T-\delta}^{T} \mathbb{E} \sum_{k \geq 1} \lambda_{k}^{-2 \rho}\left|S(s) e_{k}\right|^{2} d s\right)^{1 / 2} \\
& \leq \delta^{1 / 2}\left(\int_{T-\delta}^{T}\left|Q^{n}(s)\right|_{\mathcal{K}}^{2} d s\right)^{1 / 2}\left(\int_{T-\delta}^{T}|S(s)|_{\mathcal{K}}^{2} d s\right)^{1 / 2} \\
& \leq 2 \delta \mathbb{E} \int_{T-\delta}^{T}|S(s)|_{L(H)}^{2} d s+\frac{1}{8} \mathbb{E} \int_{T-\delta}^{T}\left|Q^{n}(s)\right|_{\mathcal{K}}^{2} d s
\end{aligned}
$$

Thus we deduce again $\frac{\text { IstimaQnunif }}{(5.27)}$, that together with $\frac{\text { StimaPUnifLyap }}{(5.5) \text { leads to prove }} \frac{\text { laprioriLyap }}{(5.2)}$

We also have that

Theorem 5.3. Under assumptions A1) - A2) - A3') equation (BSDEoperatorvalued has a unique mild solution $(P, Q)$.

Proof. The only thing to check is that following still hold:

$$
\lim _{n, m \rightarrow+\infty} \mathbb{E} \int_{T-\delta}^{T}\left|J_{n} S(s) J_{n}-J_{m} S(s) J_{m}\right|_{\mathcal{K}}^{2} d s=0
$$


Recalling that $e_{k} \in V$, for every $k \geq 1$, we have:

$$
\lim _{n, m \rightarrow+\infty}\left|J_{n} S(s) J_{n} e_{k}-J_{m} S(s) J_{m} e_{k}\right|_{\mathcal{K}}^{2} d s=0, \quad \forall k \geq 1 .
$$

Moreover

$$
\mathbb{E} \int_{T-\delta}^{T}\left|J_{n} S(s) J_{n}-J_{m} S(s) J_{m}\right|_{\mathcal{K}}^{2} d s \leq 2 \mathbb{E} \int_{T-\delta}^{T}|S(s)|_{\mathcal{K}}^{2} d s
$$

Thus by Dominated Convergence Theorem we get that $\frac{\text { convst.yap }}{5.4}$. The rest of the proof follows then identically as Theorem

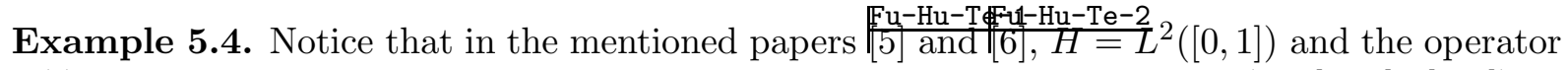
$S(t)$ is the multiplication operator by an adapted stochastic random field $H:(\Omega \times[0, T] \times[0,1]) \rightarrow$ $\mathbb{R}$ namely

$$
[S(t) e](\xi)=H(t, x) e(x), \forall e \in L^{\infty}([0,1]), \forall x \in[0,1], \quad \text { with } \quad \mathbb{E} \int_{0}^{T} \int_{0}^{1} H(t, x)^{2} d t d x<+\infty
$$

(notice that in thes case $S(t)$ is not even defined on the whole $H$ ).

Moreover the infinitesimal generator $A$ is the realization of the Laplacian in $L^{2}([0,1])$ with Dirichlet boundary conditions.

Thus we have, choosing the basis $\left\{e_{m}\right\}_{m \in \mathbb{N}}$, of eigenvectors of $A$ :

(a) $\sup _{m \geq 1}\left|e_{m}\right|_{L^{\infty}([0,1])}<\infty$.

(b) $S$ is self-adjoint and $\left|S(s) e_{m}\right|^{2}=\int_{0}^{1}\left(H^{2}(s, x) e_{m}^{2}(x) d x \leq \sup _{m \geq 1}\left|e_{m}\right|_{L^{\infty}([0,1])}^{2}|H(s, \cdot)|_{L^{2}([0,1])}\right.$

(c) $|S(s)|_{\mathcal{K}}=\sum_{k \geq 1} \sum_{m \geq 1} \lambda_{m}^{-2 \rho}\left|\left\langle S(s) e_{k}, e_{m}\right\rangle_{L^{2}([0,1])}\right|^{2}=\sum_{m \geq 1} \lambda_{m}^{-2 \rho} \sum_{k \geq 1}\left|\left\langle e_{k}, S(s) e_{m}\right\rangle_{L^{2}([0,1])}\right|^{2}$ $=\sum_{m \geq 1} \lambda_{m}^{-2 \rho}\left|S(s) e_{m}\right|_{L^{2}([0,1])}^{2} \leq\left|H^{2}(s, \cdot)\right|_{L^{2}([0,1])} \sum_{m \geq 1} \lambda_{m}^{-2 \rho} \leq \operatorname{cost}\left|H^{2}(s, \cdot)\right|_{L^{2}([0,1])}$

and Assumptions A1) A2) A3') hold.

\section{REFERENCES}

[1] A. Bensoussan, G. Da Prato, M.C. Delfour and S.K. Mitter, Representation and Control of Infinite Dimensional Systems. Vol. 1. Systems \& Control: Foundations \& Applications Birkhäuser Boston, Inc., Boston, MA, 1992.

[2] J.-M. Bismut. Linear quadratic optimal stochastic control with random coefficients. SIAM J. Contr. Optim. 14 (1976), 419-444.

[3] G. Da Prato and J. Zabczyk, Stochastic Equations in Infinite Dimensions. Cambridge University Press, Cambridge, 1992.

[4] Kai Du, Qingxin Meng, Stochastic maximum principle for infinite dimensional control systems SIAM J. Contr. Optim. to appear.

[5] M. Fuhrman, Y. Hu, G. Tessitore. Stochastic maximum principle for optimal control of SPDEs. C. R. Math. Acad. Sci. Paris, 350 (2012), n. 13-14, 683-688.

[6] M. Fuhrman, Y. Hu, G. Tessitore Stochastic Maximum Principle for Optimal Control of SPDEs. Appl. Math. Optim. 68 (2013), n. 2, $181-217$

[7] G. Guatteri, G. Tessitore. On the backward stochastic Riccati equation in infinite dimensions. SIAM J. Control Optim. 44 (2005), n. 1, pp. 159-194.

[8] Y. Hu and S. Peng, Adapted solution of a backward semilinear stochastic evolution equation. Stochastic Anal. Appl. 9 (1991), no. 4, 445-459.

[9] X.J. Li and S. Tang Maximum principle for optimal control of distributed parameter stochastic systems with random jumps. Differential Equations, Dynamical Systems, and Control Science. Lecture Notes in Pure and Appl. Math., 152 (1994), 867-890

[10] Q. Lü and X. Zhang, General Pontryagin-Type Stochastic Maximum Principle and Backward Stochastic Evolution Equations in Infinite Dimensions, Springer Briefs in Mathematics, to be published

[11] A. Lunardi Analytic semigroups and optimal regularity in parabolic problems Progress in Nonlinear Differential Equations and their Applications Vol. 16, Birkhäuser, Basel, Boston, Berlin 1995

[12] A. Pazy. Semigroups of linear operators and applications to partial differential equations. Springer-Verlag, New York, 1983.

[13] S. Peng, Stochastic Hamilton-Jacobi-Bellman Equations. Siam J. Control and Optimization. 30 (1992), $n$. 2, $284-304$. 
[14] S. Peng. Open problems on backward stochastic differential equations. Control of distributed parameter and stochastic systems (Hangzhou, 1998), Kluwer Acad. Publ., Boston, 1999.

[15] S. Tang. General linear quadratic optimal control problems with random coefficients: linear stochastic Hamilton systems and backward stochastic Riccati equations. Siam J. Control and Optimization. 42 (2003), n. $1,53-75$.

[16] J. Yong, X. Zhou, Stochastic Controls: Hamiltonian Systems and HJB Equations. Springer Verlag, Berlin, New York, 1999. 